\title{
Essais de pompage pour la nouvelle cale de construction de l'«Hellenic Shipyards» à Skaramanga
}

\author{
PAR
}

\author{
M.-F. Guiducci \\ Dr. Ing. Professeur de Mécanique des Sols \\ ì l'Université de Ancona \\ Directeur de la "Geoprogetti"
}

\author{
A. - J. Rogan \\ Dr. Ing. Chargé de cour- \\ des Travanx Maritimes \\ is l'Université Technique d'Athènes \\ Ingénieur en Chef \\ au chantier de Skaramanga
}

\section{Avant-propos}

On illustre dans cet article les essais préliminaires faits pour la réalisation du projet de la nouvelle cale de construction à Skaramanga et les essais grandeur-nature qui ont été exécutés dans la première phase de construction afin de confirmer les hypothèses de calcul, hypothèses qui étaient à la base de la possibilité même de réalisation de l'ouvrage.

L'ouvrage, qui en est maintenant au dix-huitième mois de la construction (janvier 1976, voir fig. 1) comprendra une forme de dimensions $420 \mathrm{~m}$ de long, $75 \mathrm{~m}$ de large et $9,50 \mathrm{~m}$ de profondeur, avec possibilité de prolongement jusqu'à $550 \mathrm{~m}$; l'ouvrage est destiné à la construction de navires d'un tonnage maximum de 500.000 D.W.T. et est completé par les installations nécessaires à son fonctionnement soit : quais, station de pompage, porte, voies de roulement de grues etc.

\section{Géologie et géotechnique}

Le chantier naval de 1"HELLENIC SPHIPYARDS" se situe sur la côte Est du golfe d'Eleusis à $15 \mathrm{~km}$ à l'Ouest d'Athènes et presque à $12 \mathrm{~km}$ au Nord du Pirée (voir fig. 2) au pied des collines calcaires qui constituent la formation de.base de la zone.
$1 /$ Vue aérienne du chantier (prise le 1er Janvier 1976)

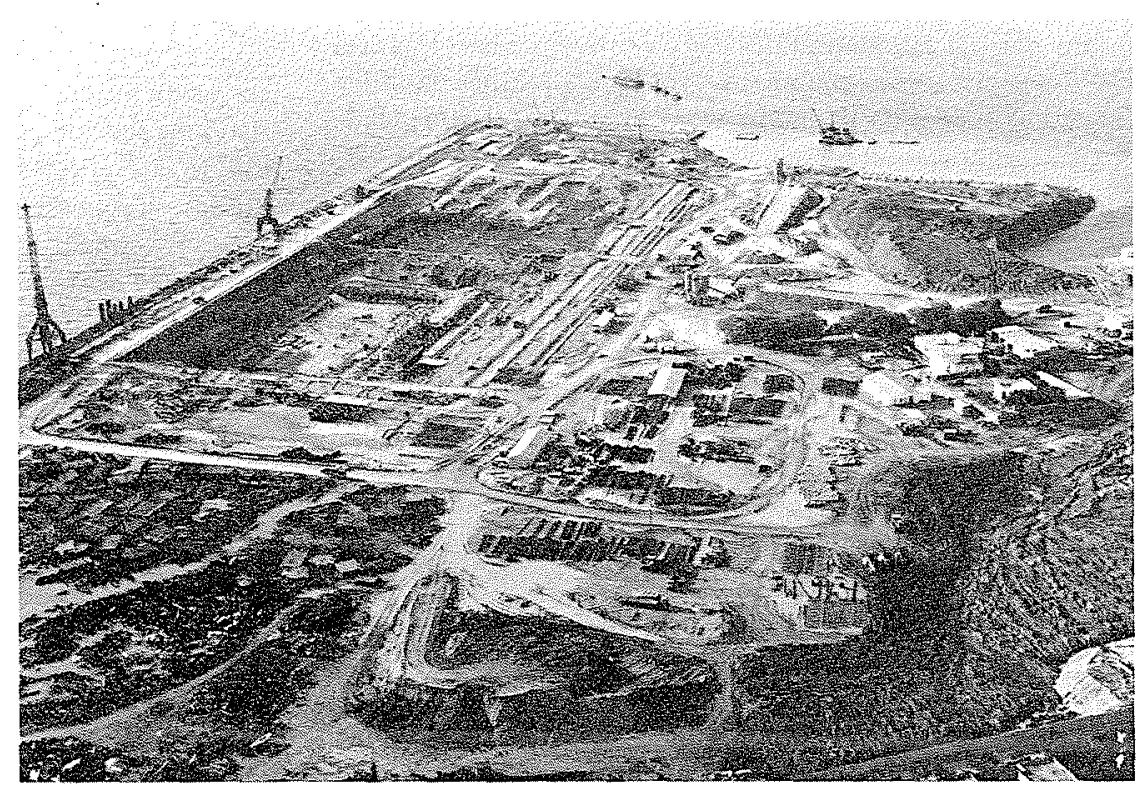




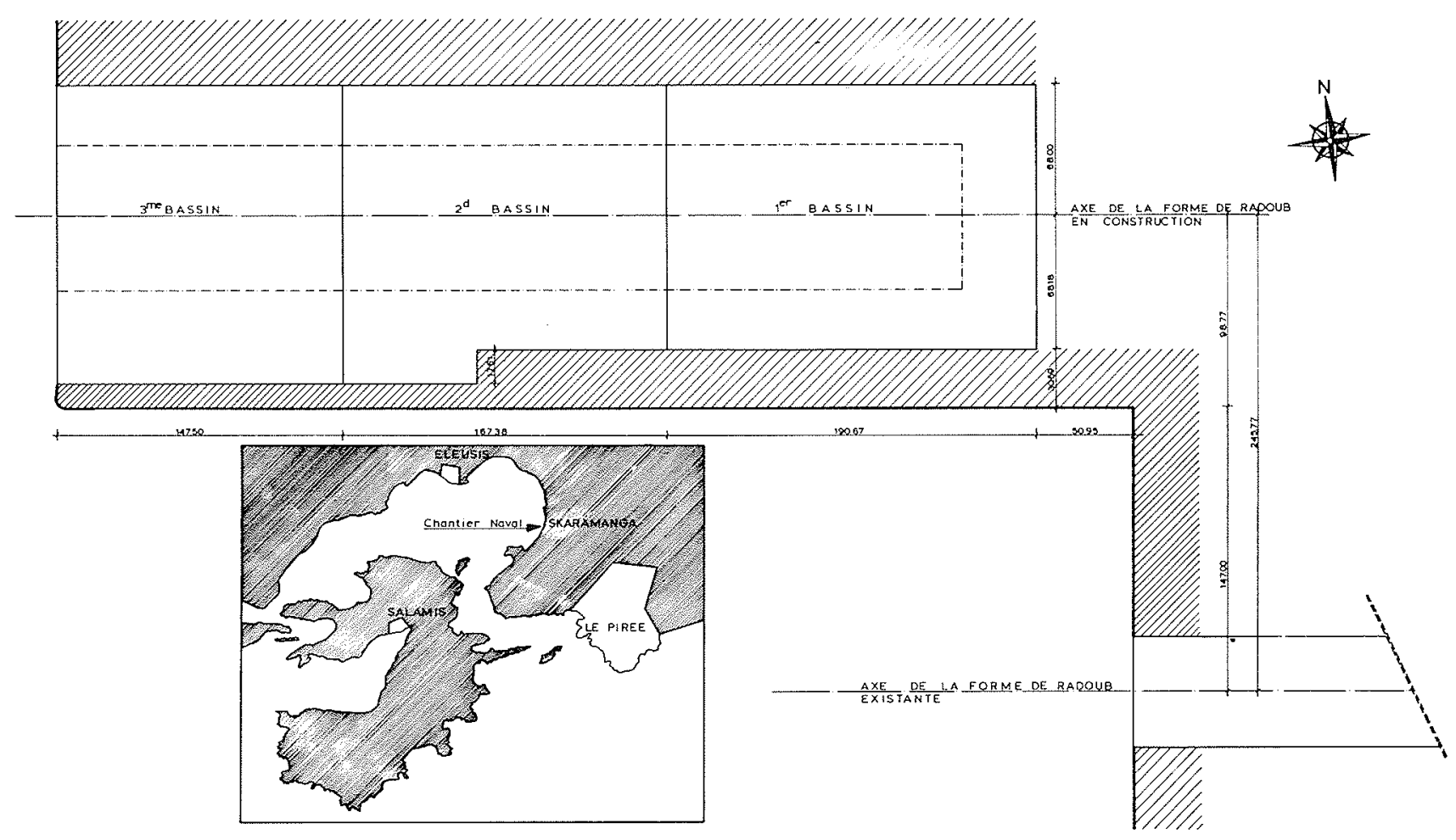

2 / Plan général - Emplacement géographique

Cette formation est extrémement fissurée et altérée, avec des vides du type karstique, d'où une perméabilité très élevée. La circulation des eaux dans ce sol est, en effet, très importante et on peut observer une nappe d'eau douce, alimentée par les collines, superposée à la nappe de la mer.

Dans la zone du chantier, et particulièrement en ce qui concerne le site du bassin en question, la formation calcaire est recouverte par une couche de matériaux de transport et de dépôt d'une épaisseur de l'ordre de 6,0 à $8,0 \mathrm{~m}$. Ces matériaux, d'origine calcaire, sont dans l'ensemble constitués par un mélange gravier, sable, limon et argile ; ils présentent une disposition à lentilles presque horizontales, dépendant de différentes phases de transport et de colmatage (voir fig. 4).

En plus, la circulation des eaux a provoqué la formation de zones cimentées toujours en forme de lentilles de faible épaisseur (ordre de grandeur $1 \mathrm{~m}$ ) mais d'extension relativement importante.

Les caractéristiques géotechniques, soit du point de vue perméabilité, soit du point de vue mécanique, se présentent, dans un terrain de ce type, extrêmement variables d'un point à l'autre bien que, dans l'ensemble, c'est-à-dire dans une échelle de dimensions rapportées à celle de l'ouvrage, on peut les considérer presque uniformes. Dans ces conditions, on peut dire qu'on devrait donner confiance plus aux essais in situ qu'aux essais de Laboratoire. En effet les essais in situ intéressent des volumes de sol relativement limités et peuvent donner des indications plus valables comparés aux résultats des essais sur des échantillons qui ne peuvent absolument pas représenter l'ensemble.

Il s'agit d'un problème d'échelle et les résultats des essais au laboratoire doivent donc être considérés sur la base de cette observation.

\section{Essais}

3.1 .

Le dossier d'Appel d'Offres, redigé par le bureau d'études "T.F. BURNS AND PARTNERS" de Londres, avait en annexe une étude des sols effectuée par la Société "KOTZIAS-STAMATOPOULOS" en 1970. Dans le but de cette étude on avait effectué 16 sondages carottés, avec mesure de la résistance au S.P.T. et à l'aide d'un nombre considérable d'essais au laboratoire sur des échantillons non remaniés on avait déterminé la classification des sols (granulométrie et limite d'Atterberg), la compressibilité et la résistance au cisaillement.

Les résultats de ce rapport sont très dispersés en relation avec la nature du terrain, mais en général, on pouvait observer qu'au dessous de la couche superficielle les caractéristiques mécaniques étaient bonnes (module de compressibilité " $E$ " entre 50 et $150 \mathrm{~kg} / \mathrm{cm}^{2}$, valeurs relativement faibles, et angle de frottement entre $25^{\circ}$ et $30^{\circ}$ ) et que le sol était constitué par des couches alternées de gravier et sable avec un pourcentage très variable d'argile et limon et avec des zones cimentées d'épaisseur allant đe quelques dizaines de centimètres à 2 mètres. On pouvait classifier les sols, selon le "Unified Soil Classification System" comme étant des types GC, GW, GP, SL -donc très variables-. Les roches calcaires fissurées ont été rencontrées à des profondeurs supérieures à $60 \mathrm{~m}$.

L'ensemble des résultats de cette campagne d'études ne pouvait pas être considéré comme suffisant pour la rédaction d'un projet d'exécution, en considérant, que pour le paramètre le plus important et déterminant pour le choix de la solution, c'est à dire la perméabilité, on ne disposait d'aucun essai valable. Seulement dans le cahier des charges on avait indiqué pour la perméabilité 
une valeur comprise entre $1,1 \times 10^{-2}$ et $4,7 \times 10^{-2} \mathrm{~cm} / \mathrm{sec}$. En plus la connaissance du module de reaction du sous-sol était indispensable pour un projet avec fondations de la dalle de fond directement appuyée.

\section{2.}

Pour les raisons exposées ci-dessus nous avons considéré comme indispensable la détermination de la perméabilité in situ et la mesure du module de réaction.

Durant les mois d'août et septembre 1971, nous avons donc exécuté une deuxième campagne d'essais, campagne qui a été effectuée par la Société "ICOS" sous la direction des techniciens de la "GEOPROGETTI".

On a exécuté 8 forages de $420 \mathrm{~m} / \mathrm{m}$ de diamètre, à percussion, et dans ces forages on a effectué des essais de perméabilité à différentes profondeurs et des essais de charge à la plaque avec mesures des déformations même à différentes profondeurs dans les forages (voir tableau 1).

Tableau 1

Mesures de perméabilité à différentes profondeurs

\begin{tabular}{|c|c|c|c|c|c|c|}
\hline SIII & SIX & SI & S III & S VII & s ZIIII & \\
\hline & & & & & & 2.50 \\
\hline & & & A) & & & 5.00 \\
\hline & 1 & 86 & $23 / 5$ & & & 7.50 \\
\hline & & $2^{45}$ & 130 & 16 & & 10.00 \\
\hline & 2.7 & 9. & $6,510,12,2$ & $2^{2}$ & & 12.50 \\
\hline & & & & $5 / 31$ & $y$ & 15.00 \\
\hline 5 & $49.0^{-2}$ & & $1 y^{3}$ & $3^{3}$ & & 17.50 \\
\hline 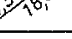 & $2^{208}$ & $8 \%$ & & & $0^{90 \%}$ & 20.00 \\
\hline & & 6."? & $5 \% 2$ & $y^{2}-21$ & & 22.50 \\
\hline 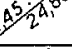 & $63^{25} / 2^{4}$ & $1 y=$ & $21 / 33^{2}$ & 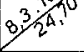 & $8 \frac{10}{2}$ & \\
\hline 6 & & $10^{6}$ & $18 / 2$ & & $2 \frac{14}{2}$ & 750 \\
\hline$\%$ & 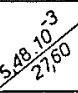 & $9 \%$ & $\frac{6}{2} 2^{1 / 9}$ & 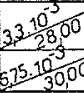 & $y$ & \\
\hline
\end{tabular}

On a exécuté 54 mesures de perméabilité et 12 mesures du module de réaction entre 1 et $7 \mathrm{~kg} / \mathrm{cm}^{2}$.

Pour la perméabilité il s'agissait de mesures de type sphérique mais essentiellement horizontales et la valeur moyenne pondérale a été $2 \times 10^{-2} \mathrm{~cm} / \mathrm{sec}$.

En considérant la formation sédimentaire des terrains et leur stratification à lentilles horizontales on peut consi- dérer comme perméabilité verticale une valeur comprise entre $1 / 5$ et $1 / 10$ de l'horizontale. En conclusion, on considère comme valeurs les plus probables

$$
K_{h}=2 \times 10^{-2} \mathrm{~cm} / \mathrm{sec} \text { et } K_{v}=0.4 \times 10^{-2} \mathrm{~cm} / \mathrm{sec}
$$

Pour la valeur du module de réaction, en considérant la transposition entre la plaque de $20 \mathrm{~cm}$ et la plaque standard de $76 \mathrm{~cm}$, les calculs donnent une valeur de $14000 \mathrm{~T} / \mathrm{m}^{3}$; en faveur de la sécurité nous avons considéré une valeur de $10000 \mathrm{~T} / \mathrm{m}^{3}$.

Une série de sondage et d'essais au laboratoire a été effectuée successivement par la Société "GEOEREVNA"; ces essais ont confirmé la nature, la classification et les caractéristiques mécaniques et de perméabilité des sols. Dans cette campagne on a aussi examiné les caractéristiques des matériaux des carrières des terres nécessaires pour remblayer la zone du chantier. L'emplacement de divers sondages est donné dans la figure 3 .

\section{Solution adoptée}

Les solutions possibles pour un bassin de construction sont en général de trois types.

- Solution à gravité : le fond et les murs du bassin doivent s'opposer par leur poids à la pression de flottement.

- Solution ancrée : les structures sont ancrées au sol, qui doit participer par son poids à équilibrer la sous-pression.

- Solution à drainage continu : la sous-pression est limitée par un drainage et un pompage continu pendant toute la vie de l'ouvrage.

Les dimensions et, en particulier, la largeur du bassin, sont telles de ne pas rendre rentable une solution à gravité, qui reste économique seulement si les murs latéraux peuvent participer efficacement à la stabilité, vis-à-vis de la sous-pression.

Une solution à drainage continu n'est d'autre part avantageuse que si le débit à pomper est limité, c'est-àdire, si la perméabilité du sol, en relation à la vitesse de l'eau dans le sol, est acceptable, et que le prix d'installation et de fonctionnement de la station de pompage le sont aussi ; dans notre cas la grandeur du débit aurait conduit au choix d'une très importante station de pompage, avec des vitesses de l'eau dans le sol dangereuses en ce qui concerne le transport des grains fins.

Nous avons choisi la solution ancrée comme la plus valable et économique, en prévoyant le rabattement de la nappe seulement pendant la construction.

Pour rendre possible ce rabattement on a prévu une ceinture de parois moulées, divisées en trois secteurs (bassins), pour clôturer latéralement les zones à excaver, en laissant seulement à pomper l'eau d'infiltration du fond (voir fig. 2).

Le système prévoit à l'avance le remblayage complet de la zone du bassin, successivement la construction des parois moulées et des ancrages en béton précontraint à partir du remblai. 


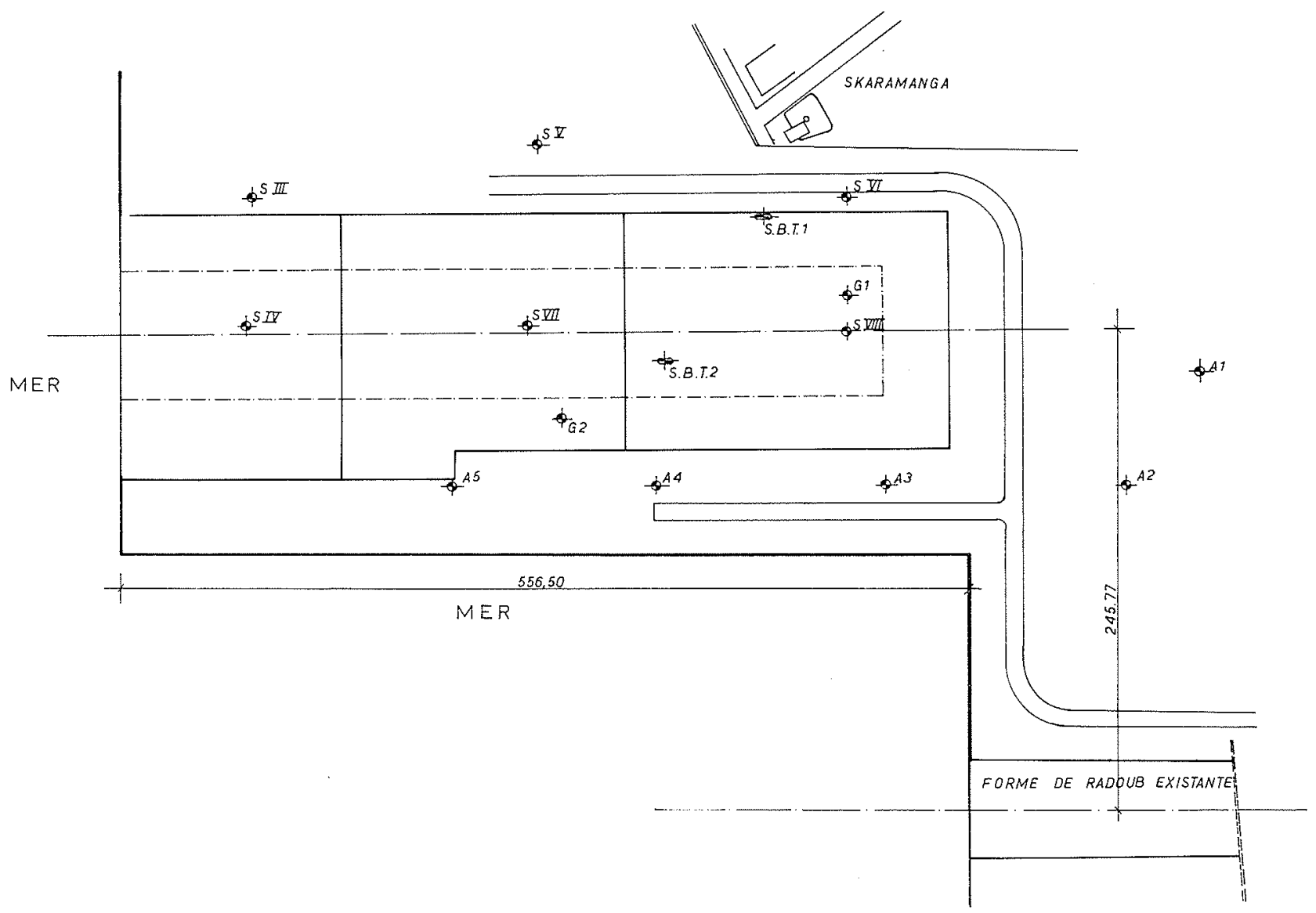

$3 /$ Sondages

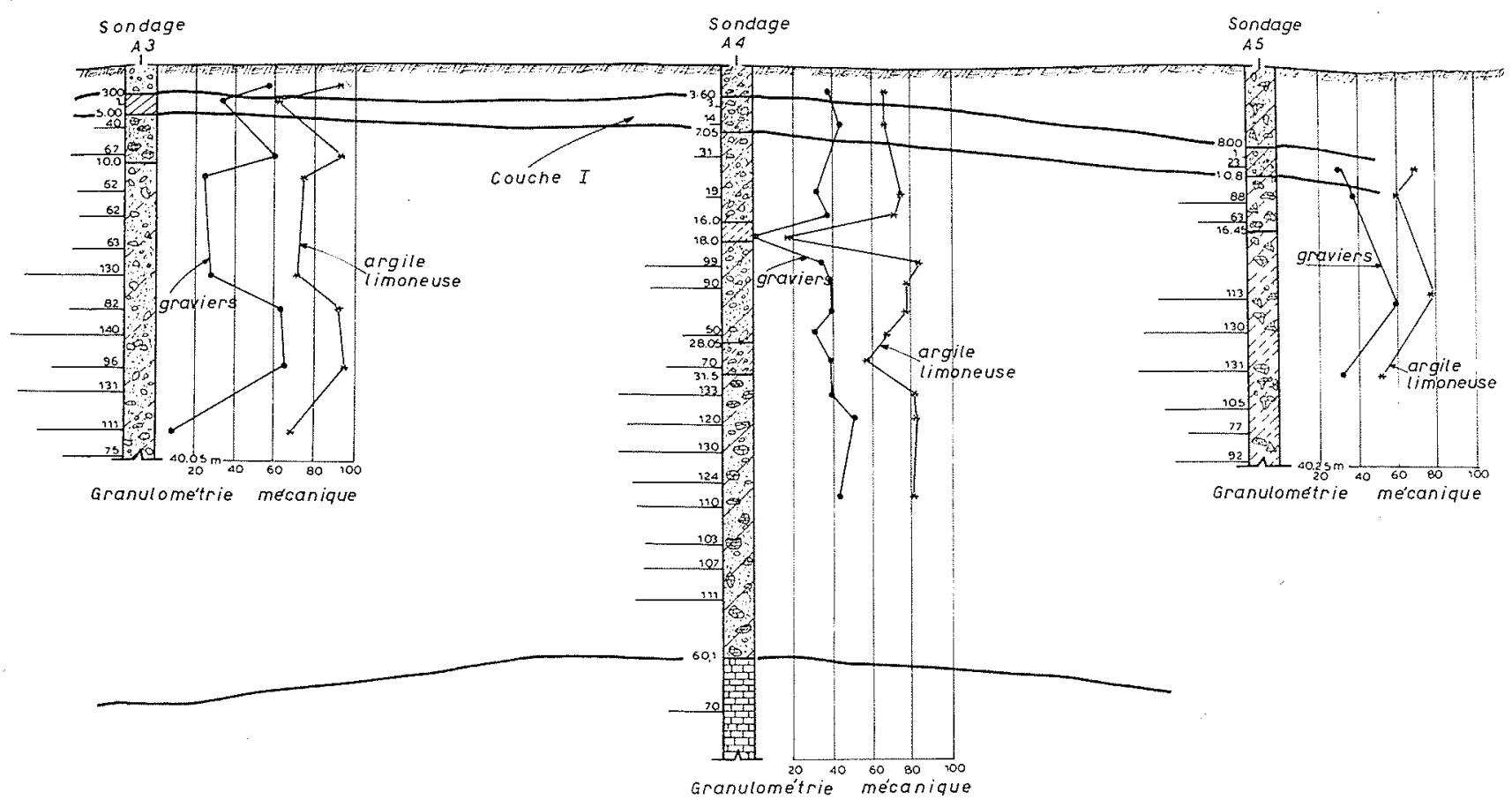

4/ Coupe du sous-sol, d'après les sondages (en relation avec la figure 3 ). 
Dans le même temps on procède à la construction des puits, à l'intérieur de la ceinture, pour le rabattement de la nappe. Ces puits doivent avoir une profondeur limitée et être, par conséquent, de grand diamètre et très diffusés dans le but de ne pas capter les eaux en profondeur et de réduire au maximum la vitesse locale d'écoulement.

Les parois moulées ont donc une profondeur de $25 \mathrm{~m}$ et les puits de $16 \mathrm{~m}$ à partir de la côte zéro (voir fig. 10). En considérant que le niveau de l'excavation est prévu à la côte de $-12,5$, dans la phase finale de leur fonctionnement, les puits ont une profondeur efficace de $3,5 \mathrm{~m}$ seulement.

La validité du système est liée au débit à pomper, débit pour lequel une erreur même de $100 \%$ est fort probable en relation aux approximations des mesures de perméabilité effectuées.

Pour cette raison on a décidé d'effectuer dans la première zone (dimensions 190,67 x 136,18 m) un essais général de rabattement, avant de construire les ancrages, afin de vérifier si le pompage était vraiment possible et si les installations pour le rabattement (puits, pompes, etc.) prénues au projet étaient conformes aux nécessités réelles. Pour le cas d'impossibilité de pompage on avait déjà prévu une solution à gravité.

\section{Calculs du débit à pomper}

La disposition "à tapis" des puits permet de considérer le pompage comme uniformément distribué à la cote finale de l'excavation $(-12,5)$. Avec les valeurs de perméabilité déjà exposées $\left(K_{h}=2 \times 10^{-2} \mathrm{~cm} / \mathrm{sec} ; K_{v}=0,4 \times 10^{-2}\right.$ $\mathrm{cm} / \mathrm{sec}$ ) on peut calculer la valeur du débit par trois différentes méthodes:

a) à l'aide du tracé du réseau d'infiltration

b) à l'aide de la formule de Darcy en considérant l'écoulement vertical dans le terrain compris entre les parois moulées verticalement, et entre les cotes -25 et $-12,5$ horizontalement

c) à l'aide des graphiques de Neyrpic.

\subsection{Réseau d’infiltration}

Pour le calcul, du débit on trace les lignes de courant et les équipotentielles, dans l'hypothèse d'un fond imper- méable à $80 \mathrm{~m}$ de profondeur, et en considérant une perméabilité uniforme (horizontale et verticale) $K=2 \times 10^{-4} \mathrm{~m} / \mathrm{sec}$. L'hypothèse du fond à $-80 \mathrm{~m}$ s'identifie pratiquement à celle d'un terrain perméable allant jusqu'à une profondeur infinie. C'est ainsi que dans la figure 5 on considère l'excavation allant jusqu'à $-12,50$ et la couche imperméable à $-80 \mathrm{~m}$.

Le débit total pour tout le bassin est ainsi calculé à $1,73 \mathrm{~m}^{3} / \mathrm{sec}$.

Pour les successives phases de pompage les débits résultent :

\begin{tabular}{|l|c|c|c|}
\hline Bassin & $\begin{array}{r}\text { Débit } \\
\mathrm{m}^{3} / \mathrm{sec}\end{array}$ & No. puits & $\begin{array}{c}\text { Débit/puits } \\
1 / \mathrm{sec} .\end{array}$ \\
\hline 1 & 0,86 & 77 & 11 \\
2 & 0,80 & 81 & 10 \\
$1+2$ & 1,30 & - & - \\
3 & 0,82 & 56 & 15 \\
$2+3$ & 1,23 & - & - \\
\hline
\end{tabular}

\subsection{Formule de Darcy}

On considère que l'infiltration globale provient d'une couche filtrante d'une surface totale de :

$$
A=505 \times 135=68175 \mathrm{~m}^{2}
$$

d'une épaisseur de :

$$
S=25-12,5=12,5 \mathrm{~m}
$$

et d'un coefficient de perméabilité verticale :

$$
K_{v}=0,4 \times 10^{-4} \mathrm{~m} / \mathrm{sec}
$$

En appliquant la formule de Darcy on obtient comme débit global :

$$
\begin{aligned}
Q=K_{v} \times i \times A & =K_{v} \times \frac{\Delta H}{S} \times A= \\
& 0,4 \times 10^{-4} \times \frac{12,5}{12,5} \times 68175=2,72 \mathrm{~m}^{3} / \mathrm{sec}
\end{aligned}
$$

\subsection{Graphique de Neyrpic}

Le calcul théorique appliqué dans ce paragraphe est illustré en détails dans la Référence [4].

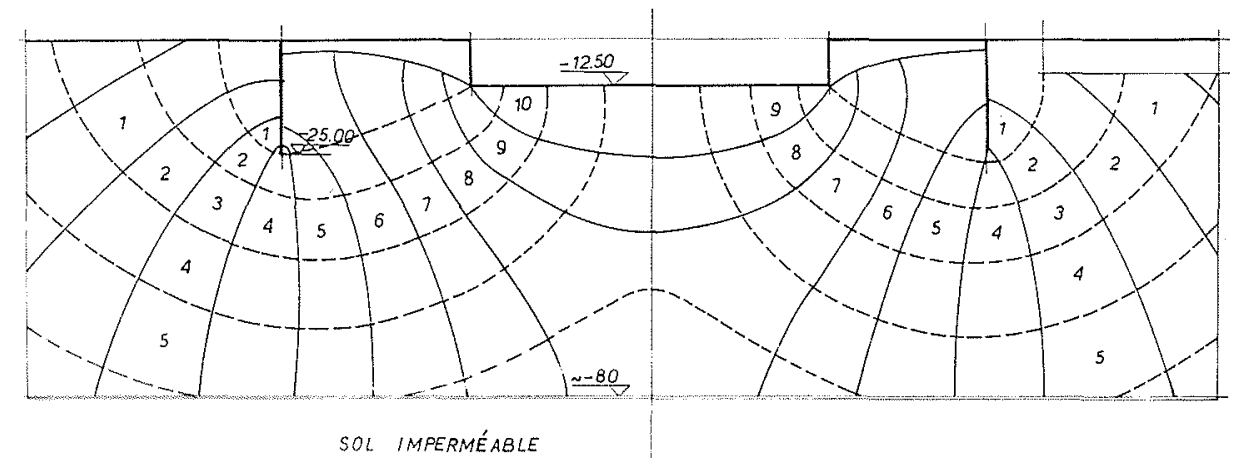

$5 /$ Maille des lignes equipotentielles 


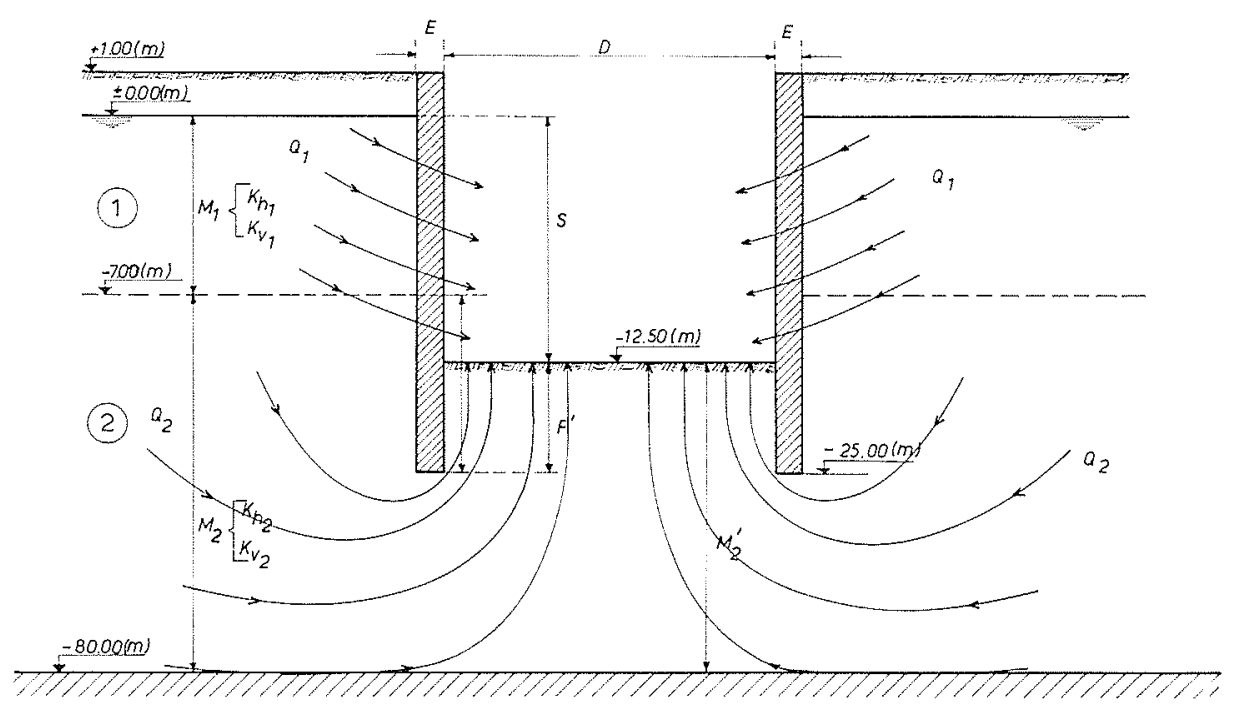

6/ Notations pour le calcul de débit (présentation schématique)

La figure 6 définit les notations utilisées dans le cas du sol de Skaramanga. La couche "I" est relativement faible ( 7 mètres d'épaisseur) et barrée par un écran (paroi moulée) ancré dans une couche " 2 ", moins perméable. On décompose le débit global (Q) à pomper en deux parties :

$Q_{1}=$ débit de filtration à travers l'écran

$Q_{2}=$ débit de contournement sous l'écran

Cette estimation est plus détaillée que celle décrite au paragraphe 5.2. On fait toujours l'hypothèse que la couche imperméable se trouve à la profondeur de $-80 \mathrm{~m}$ et le niveau d'excavation dans le bassin, à $-12,50 \mathrm{~m}$. C'est ainsi que la couche "2" a une épaisseur de $73 \mathrm{~m}$.

Les coefficients de perméabilité correspondants sont les suivants :

- Pour la couche supérieure, formée de matériaux de transport et de dépôt :

$$
K_{h_{1}}=4,5 \times 10^{-2} \mathrm{~cm} / \mathrm{sec}
$$

- Pour la couche inférieure, composée des formations plus anciennes des alluvions:

$$
\begin{aligned}
& K_{h_{2}}=2 \times 10^{-2} \mathrm{~cm} / \mathrm{sec} \\
& K_{v_{2}}=0,4 \times 10^{-2} \mathrm{~cm} / \mathrm{sec}
\end{aligned}
$$

\subsubsection{Calcul du débit $Q_{1}$}

En admettant que le coefficient de perméabilité à travers les parois moulées en béton soit :

$$
K_{h}=10^{-8} \mathrm{~cm} / \mathrm{sec}=10^{-10} \mathrm{~m} / \mathrm{sec}
$$

on calcule le débit de filtration par $m^{2}$ de paroi moulée à l'aide de la formule :

$$
\begin{aligned}
q_{1}=K \times H_{m} \times i=10^{-10} \times 3,50 \times \frac{3,50}{0,60} \\
. \quad=20,42 \times 10^{-10} \mathrm{~m}^{3} / \mathrm{sec} / \mathrm{m}^{2}
\end{aligned}
$$

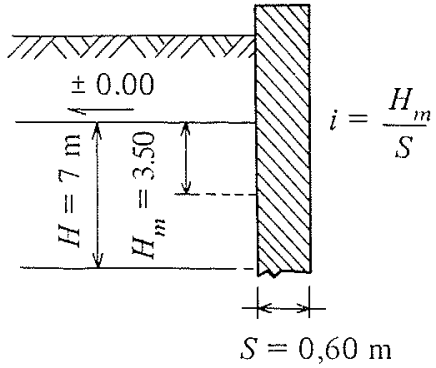

La surface de filtration est

$$
F=2 \times(505+135) \times 7=8960 \mathrm{~m}^{2}
$$

On a alors :

$Q=q_{1} \times F=20,42 \times 8960 \times 10^{-10}=0,000018 \mathrm{~m}^{3} / \mathrm{sec}$ débit pratiquement négligeable

\subsubsection{Calcul du débit $Q_{2}$}

Les formules sont écrites pour le débit par unité de périmètre $q_{2}=Q_{2} \mid P$

Le rabattement est divisé en trois parties:

$S^{\prime}=$ pertes de charge pour les lignes de courant descendantes

$S$ " = pertes de charge pour l'écoulement horizontal sous la largeur $E$ de l'écran

$S^{\prime \prime \prime}=$ pertes de charge pour les lignes de courant ascendantes

Le débit $q_{2}$ est une fonction de $k_{h_{2}}, k_{v_{2}}, M_{2}, M_{2}^{\prime}$, $F, F^{\prime}, E, D$ (ou plus précisement de tout le tracé de l'écran) notations données à la figure 6 .

On fait l'hypothèse que tout le débit $Q_{2}$ vient de la nappe 1, qu'au-dessous du niveau $-80,00$ la couche est imperméable (c'est-a-dire que le débit $Q_{3}$, selon [4], est nul et par suite $Q_{3} / Q_{2}$ complètement négligeable) et que la dimension $D$ est très grande. 
On calcule premièrement la relation :

$$
\begin{aligned}
3 M_{2}^{\prime} \sqrt{K_{h_{2}} / K_{\nu_{2}}}=3 \times 67,5 \times \sqrt{2 \times 10^{-2} / 0,4 \times 10^{-2}} \\
=128,07 \mathrm{~m}
\end{aligned}
$$

La dimension plane minimale est :

$$
D=135 \mathrm{~m}>128,07 \mathrm{~m}
$$

en même temps, l'épaisseur $E=0,60 \mathrm{~m}$ de la paroi moulée est relativement négligeable, en la comparant à la dimen$\operatorname{sion} D=135 \mathrm{~m}$

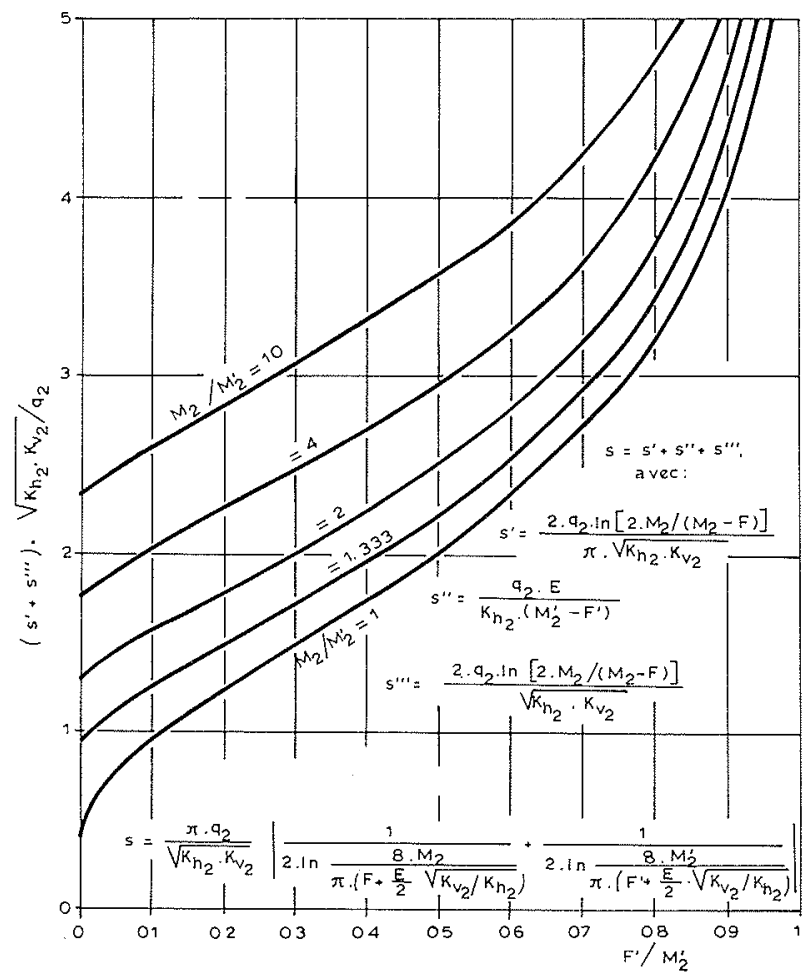

$7 / q_{2}$ pour $D>3 M_{2}^{\prime} \times \sqrt{K_{h_{2}} / K_{V_{2}}}$ page 34 , de l'article de MM. J. Cardinal et E. de Cazenove.

Par suite on utilisera la transposition du graphique Neyrpic (voir fig. 7)

$$
\left.\begin{array}{c}
\frac{M_{2}}{M_{2}^{\prime}}=\frac{73}{67,5}=1.08 \\
\frac{F^{\prime}}{M_{2}^{\prime}}=\frac{12,50}{67,50}=0,185
\end{array}\right\} \begin{array}{r}
\frac{S^{\prime}+S^{\prime \prime \prime}}{q_{2}} \times \sqrt{K_{h_{2}} \times K_{v_{2}}} \\
=1,21 \\
S=S^{\prime}+S^{\prime \prime}+S^{\prime \prime \prime}=12,50 \mathrm{~m}
\end{array}
$$

et par suite

$$
S^{\prime}+S^{\prime \prime \prime}=12,50-S^{\prime \prime}
$$

En remplaçant (2) à (1)

$$
\frac{12,50-S^{\prime \prime}}{q_{2}} \times \sqrt{K_{h_{2}} \times K_{v_{2}}}=1,21
$$

mais la valeur de $S^{\prime \prime}$ est donnée par

$$
S^{\prime \prime}=\frac{q_{2} \times E}{K_{t_{2}}^{\prime}\left(M_{2}^{\prime}-F^{\prime}\right)}
$$

en introduisant (4) à (3)

$$
\frac{12.50-\frac{q_{2} \times E}{K_{h_{2}}\left(M_{2}^{\prime}-F^{\prime}\right)}}{q_{2}} \sqrt{K_{h_{2}} \times K_{v_{2}}}=1,21
$$

et

$$
\begin{gathered}
q_{2}=9,2 \times 10^{-4}\left[\mathrm{~m}^{3} / \mathrm{sec} / \mathrm{m} .1 .\right] \\
Q_{2}=P \times q_{2}=[2 \times(135+505)] \times 9,2 \times 10^{-4} \\
=1.1776 \mathrm{~m}^{3} / \mathrm{sec}
\end{gathered}
$$

Le débit global est alors:

$$
Q=Q_{1}+Q_{2}=1,177618 \simeq 1,18 \mathrm{~m}^{3} / \mathrm{sec}
$$

\section{4 .}

Le débit maximum de chaque puits ne peut pas excéder une certaine valeur, en fonction de la perméabilité, afin d'éviter le transport des particules fines du sol. Ce débit maximum, en phase finale, en considérant des puits de $1,20 \mathrm{~m}$ de diametre et de $3,50 \mathrm{~m}$ de profondeur, audessous de $-12,50$ (rayon moyen : $2 \mathrm{~m}$ ), est :

$$
\begin{aligned}
q=4 \times R \times K \times h=12,60 \times 2 \times 2 \times 10^{-4} \times \frac{3,50}{2} \\
=8,51 / \mathrm{sec}
\end{aligned}
$$

Etant donné que le débit calculé est supérieur à cette quantité, une partie de l'eau sera pompée à travers le drainage superficiel.

\section{Installation d'essais de rabattement}

L'essai (échelle nature) de rabattement de la nappe d'eau a été effectué dans le premier bassin (voir fig. 8) qui représente environ $1 / 3$ de la cale. L'extrémité Est de la câle sera construite dans ce bassin. Les résultats de cet essäi doivent être considérés comme représentatifs pour les trois bassins ; cette considération est appuyée par le fait que les deux autres sont construits en majeure partie à travers un remblai qui est plus homogène du sol naturel.

Ce premier bassin est protégé par des parois moulées, allant jusqu'à une profondeur de $-25 \mathrm{~m}$. Les dimensions du bassin sont : 190,67 x 135,40 m (voir fig. 9). Les parois moulées, "Nord" et "Sud" sont en béton armé, et celles "Est" et "Ouest" en béton plastique : ciment et bentonite.

Les parois en béton armé seront utilisées pour les fondations des rails des grues et des portiques du chantier naval.

\subsection{Puits}

77 puits, d'un diamètre interne de $0,70 \mathrm{~m}$, et d'une profondeur de $17 \mathrm{~m}$ (voir fig. 9 et 10) ont éte construits à l'aide des éléments de tuyaux préfabriqués en béton armé (perforés entre $-8,20$ et $-15,20 \mathrm{~m}$ ) qui sont entourés d'un filtre sable-graviers, d'une épaisseur moyenne de $0,25 \mathrm{~m}$. 


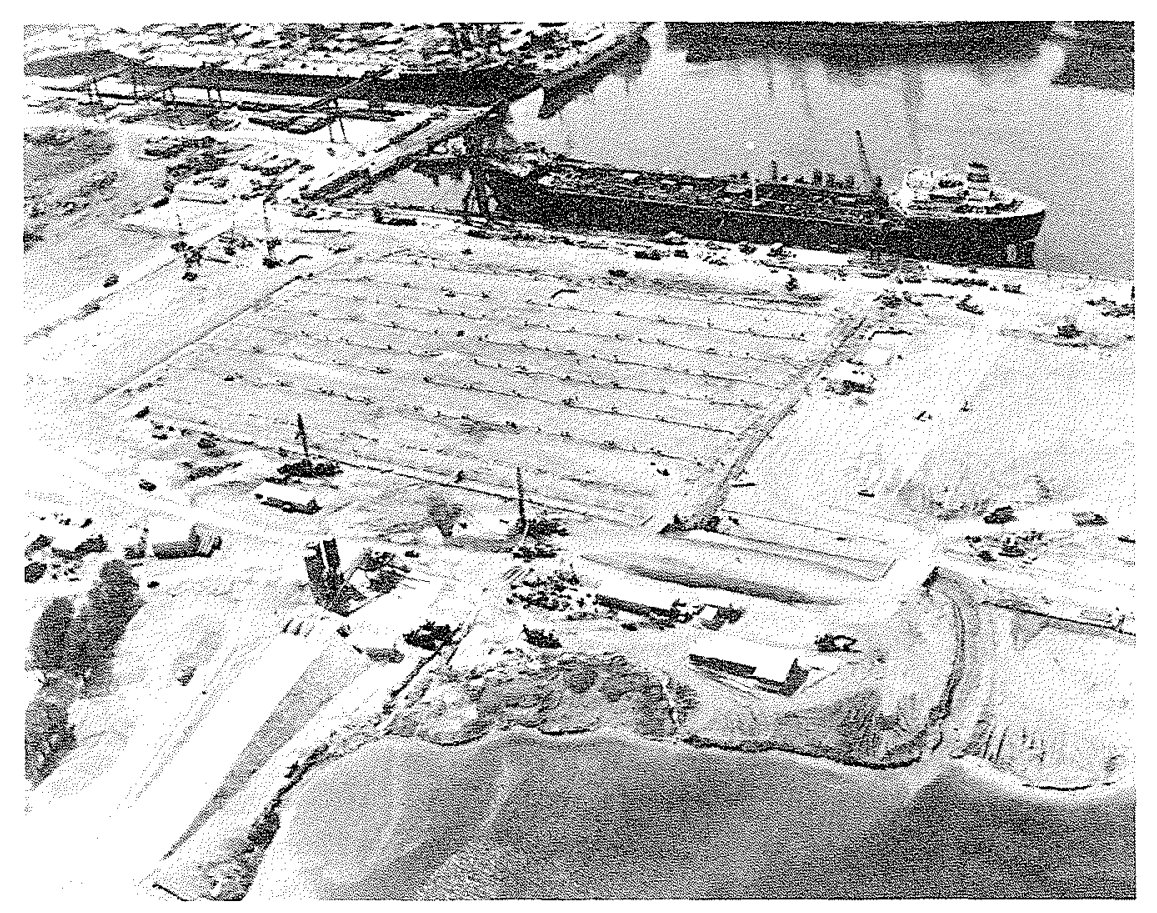

8/Vue aérienne du 1er bassin pendant l'essai.

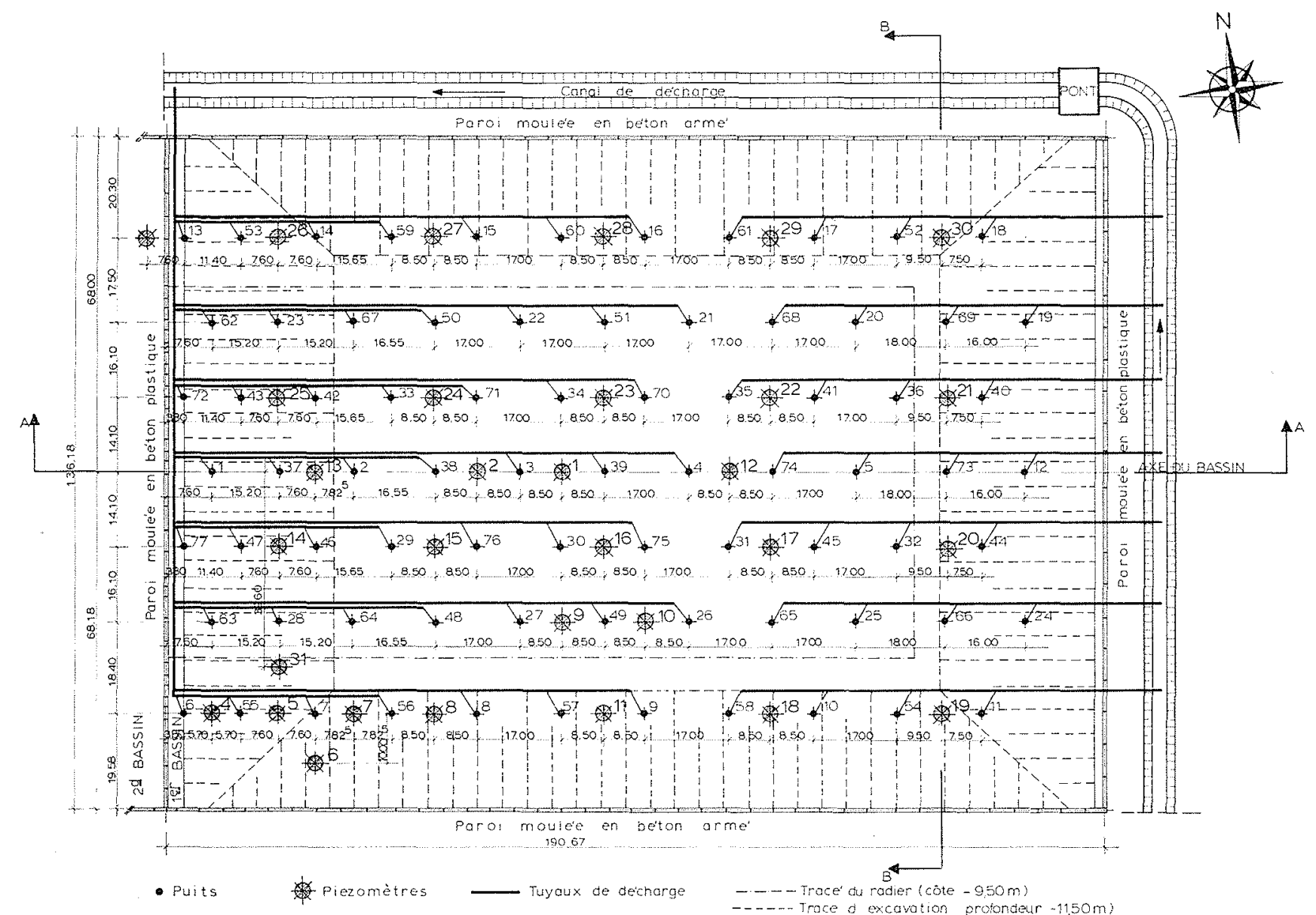

9/Plan du 1er bassin avec installations de pompage et mesures. 

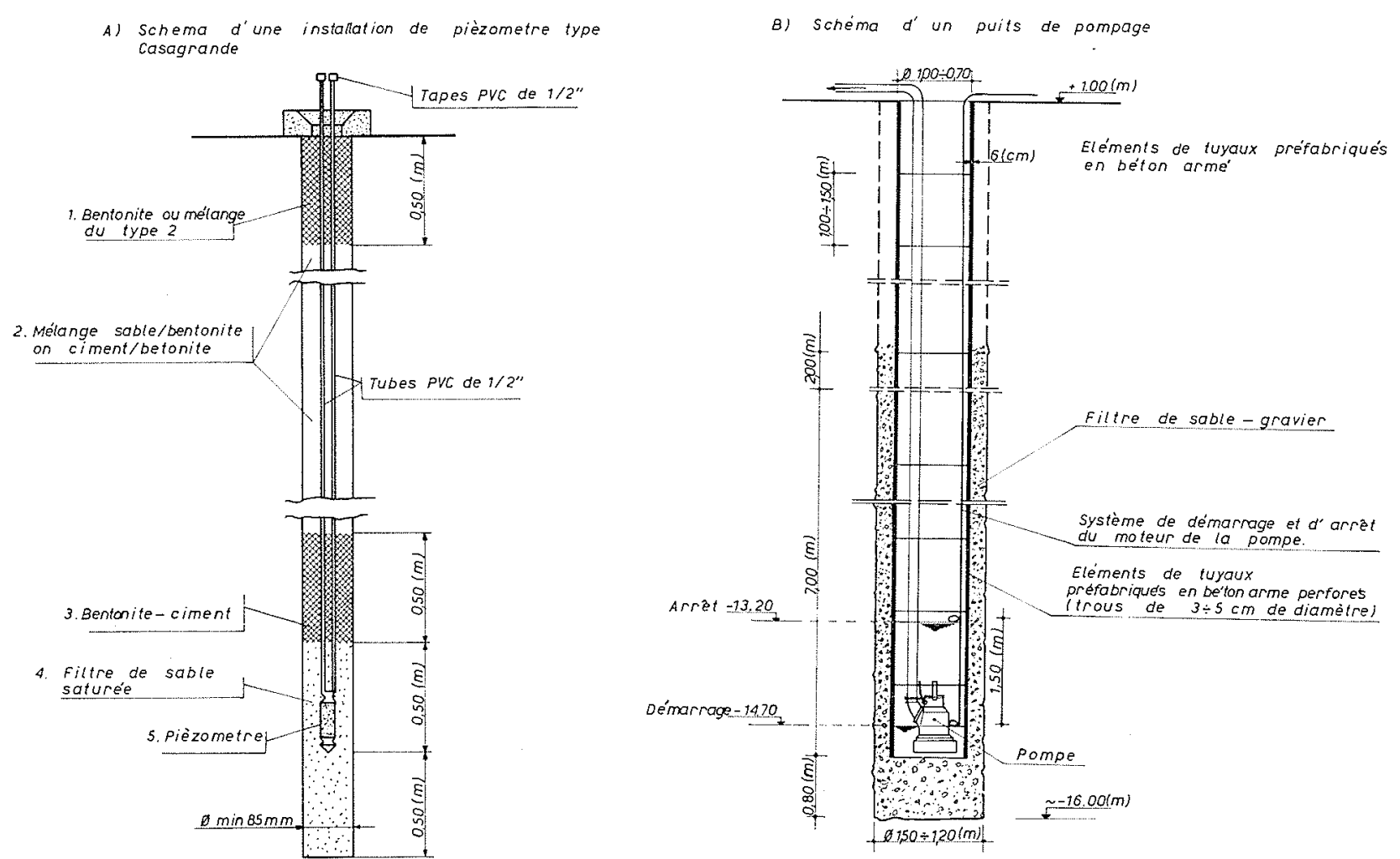

10/ Coupes d'une piézomètre et d'ún puits

Les puits sont équipés de pompes sous-marines FLYGT. Elles sont du type B2102/MT, 7CV avec moteur ayant les caractéristiques : 2850 (tours $/ \mathrm{min}$ ), 380 (V), $50(\mathrm{HZ})$ et un tuyau de sortie de 4 pouces (ou $100 \mathrm{~mm}$ ). Les tuyaux des pompes étaient raccordés à d'autres tuyaux plastiques, d'un diamètre de $200 \mathrm{~mm}$, qui déchargeaient les eaux pompées dans un canal périphérique (voir fig. 9).

Tous les pompes étaient équipés d'un système de flotteurs interrupteurs automatiques pour assurer la constance du niveau de l'eau dans les puits.

Le débit maximum pompable est supérieur au débit calculé théoriquement de $100 \%$, marge absolument nécessaire pour couvrir, à part les approximations de calcul, les accidents de fonctionnement des pompes.

\subsection{Pièzomètres}

Trente pièzomètres type Casagrande ont été disposés dans le premier bassin et un dehors (voir fig. 9 et 10). Ils allaient jusqu'à une profondeur de $-15,50 \mathrm{~m}$. Les forages avaient un diamètre de $85 \mathrm{~mm}$ et le pièzomètre était installé à une profondeur de $-15 \mathrm{~m}$ dans un filtre de sable saturé (voir fig. 10). Le pièzomètre était relié à la surface par deux tubes en PVC de $1 / 2$ " de diamètre. Le reste du forage était rempli avec un mélange de sable et bentonite. Deux tapes, en PVC également, bouchaient les tuyaux et les protégaient.

Les niveaux pièzomètriques étaient mesurés avec des instruments éléctriques SEO2A de la Société S.I.S. Chaque instrument se compose d'une boîte de mesures (qui est alimentée de deux piles de $4,5 \mathrm{~V}$ ), d'un rouleau avec une corde graduée de $100 \mathrm{~m}$ de longueur, au bout de laquelle se trouve la sonde. La sonde a un diamètre de $12 \mathrm{~mm}$ et peut traverser facilement les tubes, en PVC, du pièzomètre.

Le niveau de l'eau dans les puits était mesuré avec une sonde manuelle avec une approximation de $5 \mathrm{~cm}$.

\section{Procédure de l'essai}

L'essai a commencé le $26 / 3 / 75$ et s'est poursuivi jusqu'au $8 / 4 / 75$. Pendant ces 14 jours on a effectué les mesures suivantes :

\subsection{Mesures des niveaux pièzomètriques}

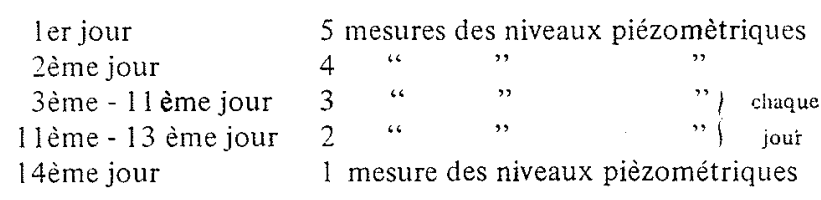

On a tracé le réseau des lignes isopièzomètriques (voir fig. $11,12,13,14$ ) en faisant lhypothèse que les 31 mesures étaient faites simultanément. Cette hypothèse simplifiante n'introduisait pas une erreur significative, parce que le pompage était continu et le rabattement de la nappe relativement lent.

Les mesures effectuées avec les piézomètres ne fournissaient que la hauteur piézométrique à la côte $-16 \mathrm{~m}$ et pas le niveau de la surface libre de la nappe. Pour obtenir ce niveau il fallait soustraire de la valeur mesurée dans les pièzomètres la hauteur correspondante de la perte hydrodynamique évaluée entre 10 et $20 \%$ de la colonne d'eau superposée (ce qui équivalait à 0,60 à $1,20 \mathrm{~m}$ ).

L'ensemble des mesures est présenté dans le tableau 2. 


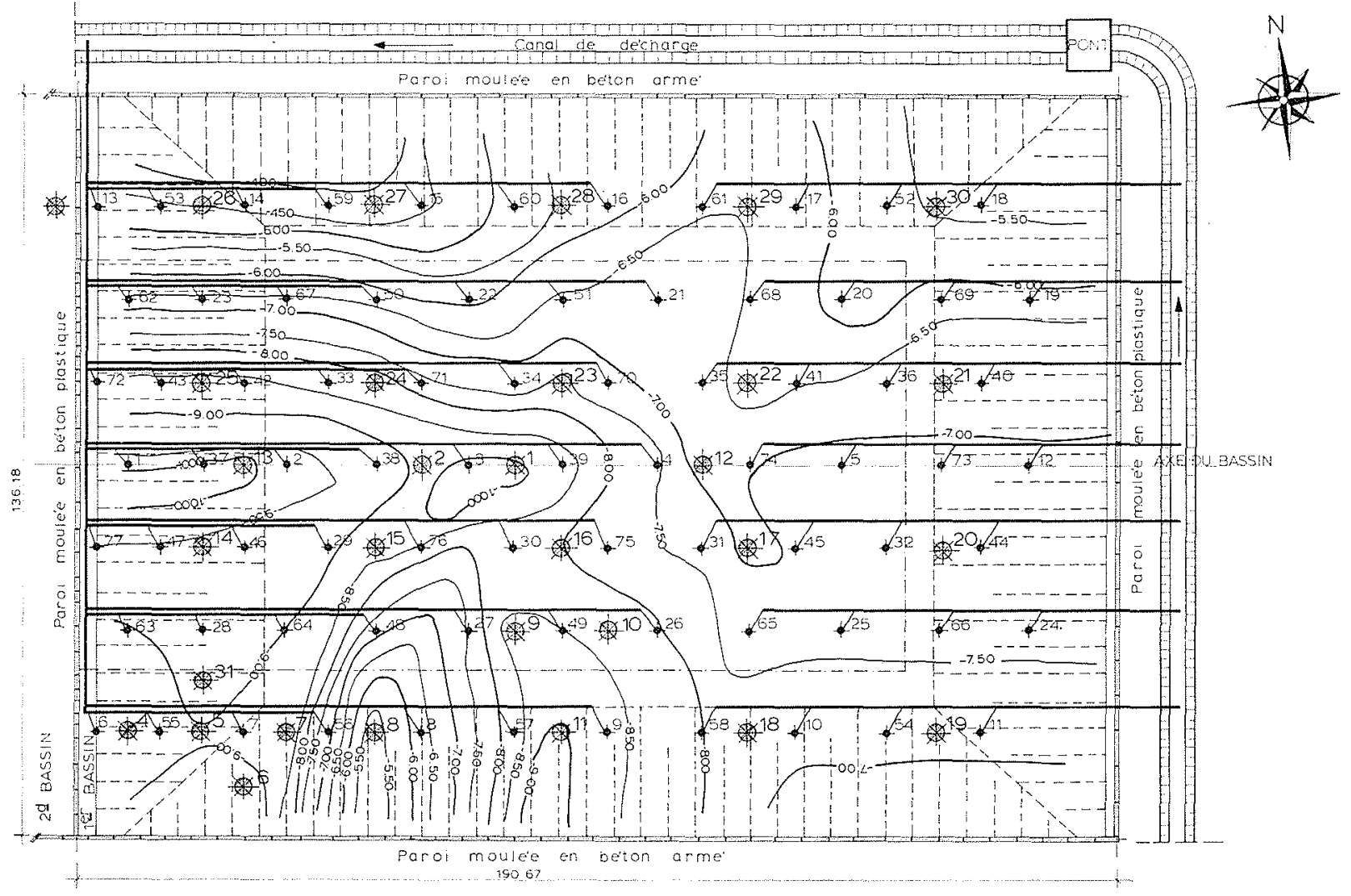

- Puits Piezomètres Tuycux de decharge - - Trace du radier (cote $-9,50 \mathrm{~m}$ )

11/ Lignes isopiézométriques du 26/3/75 à 09.30 heures

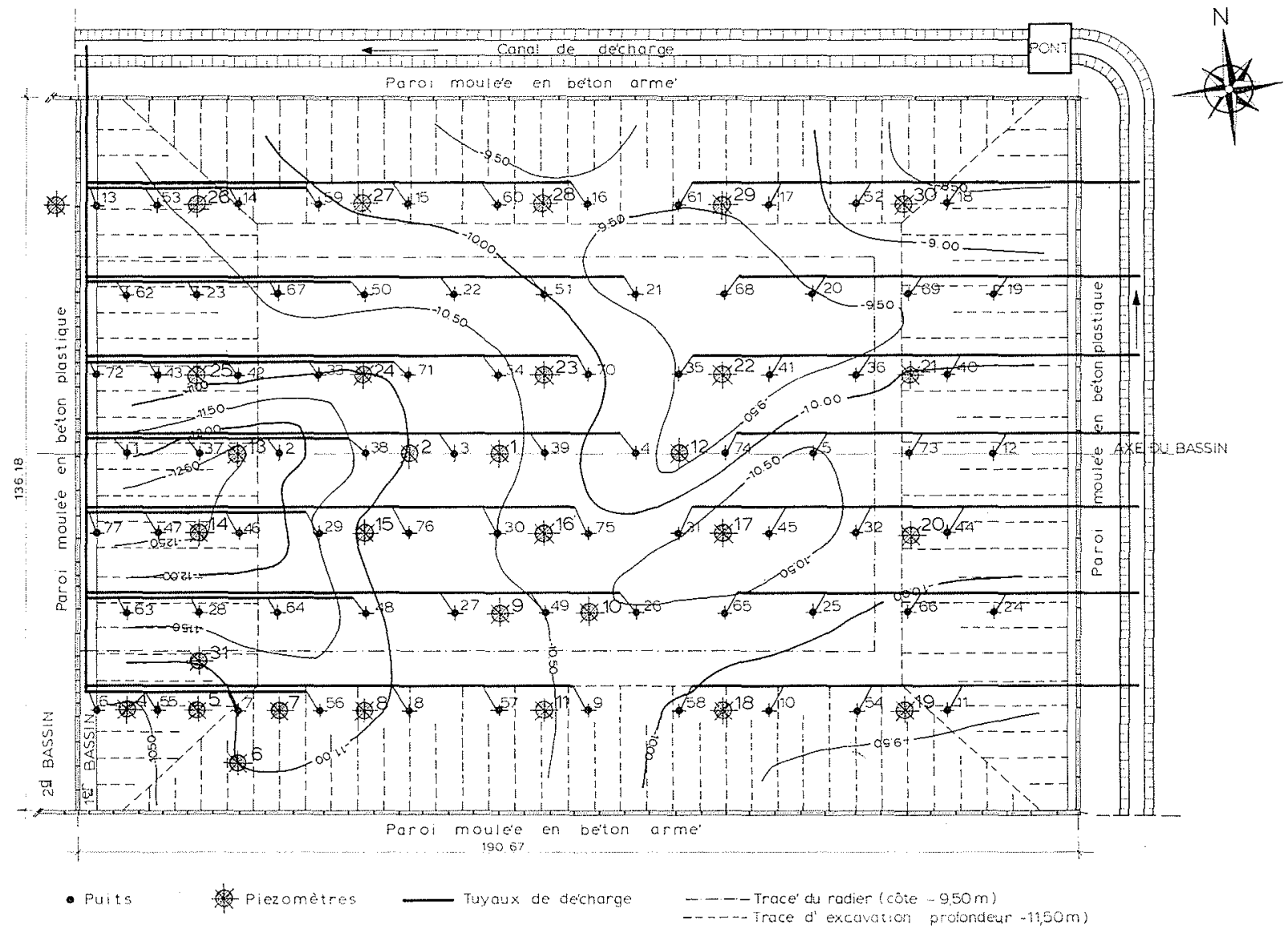

12/ Lignes isopiezométriques du 30/3/75 à 08.00 heures 


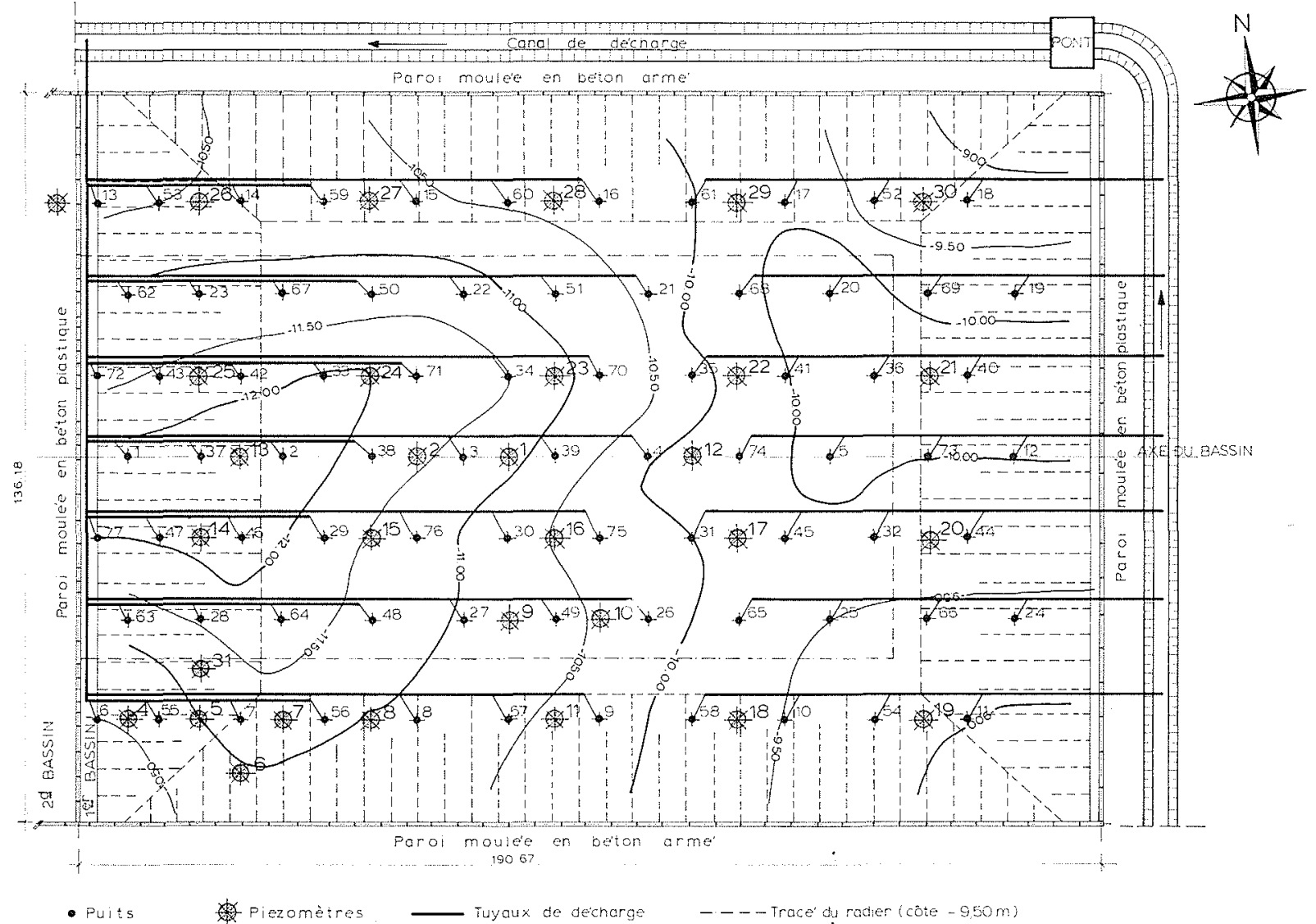

13/ Lignes isopiézométriques du 4/4/75 à 08.00 heures

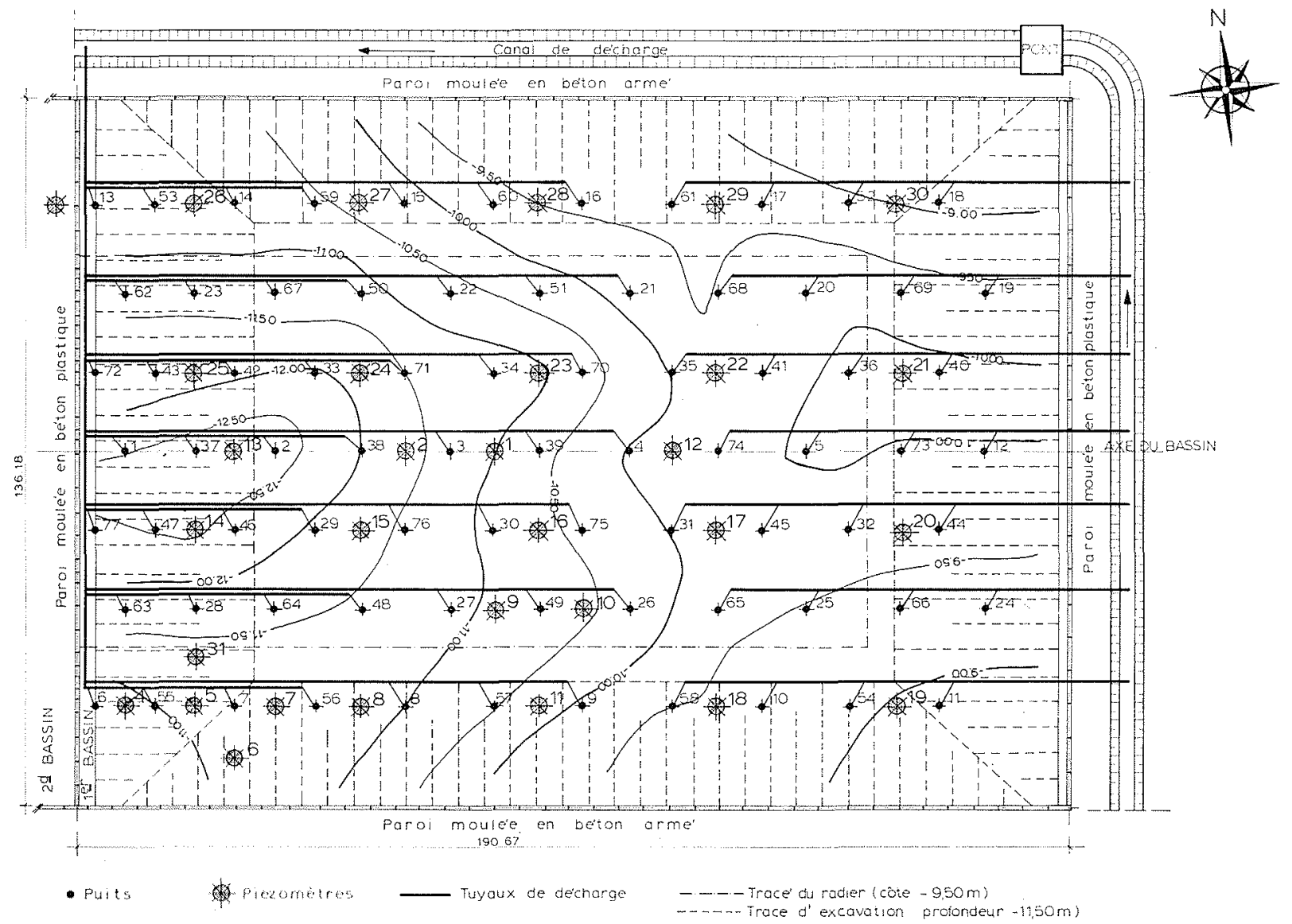

14 Lignes isopiézométriques du 8/4/75 à 10.00 heures 
Tableau 2

Mesures piézométriques

\begin{tabular}{|c|c|c|c|c|c|c|c|c|c|c|c|c|c|c|c|c|c|c|c|c|c|c|c|c|c|c|c|c|c|c|c|c|}
\hline \multicolumn{2}{|c|}{ MÉSURES NUMERO } & 1 & 2 & 3 & 4 & 5 & 6 & 7 & 8 & 9 & 10 & 11 & 2 & 3 & 14 & 5 & 16 & 7 & 18 & 19 & 20 & 21 & 22 & 123 & 24 & 25 & 26 & 27 & 28 & 29 & 30 & \\
\hline IE & HEURES & & & & & & & & & & & & & & & & & & & & & & & & & & & & & & & \\
\hline 264 & & & 82 & 75 & & -9.02 & 11 & 32 & 20 & & 37 & 15 & 96 & 06 & 48 & 85 & 3.02 & 96 & 68 & 64 & $1 ?$ & 92 & -639 & 752 & 26 & 69 & 3 & 67 & & 647 & & \\
\hline .." & & & 22 & 10 & & -918 & .06 & E1 & $\therefore$ & 50 & 87 & 6 & 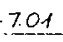 & $0.04 !$ & 072 & -930 & 8.15 & -6.96 & 53 & $0:$ & 32 & 747 & -6.78 & -868 & 을 & 1922 & 846 & 779 & 50 & $6 \varepsilon 2$ & 45 & \\
\hline & & 10 & {$[-057]$} & $118^{\circ}$ & 8.02 & -9.56 & 947 & 980 & 9.57 & 73 & 15 & $\infty$ & 152 & 897 & 994 & 939 & 8 & 739 & 680 & $7 \leq 4$ & 793 & 794 & -731 & 1918 & 1000 & 1492 & -908 & 859 & 340 & {$[738$} & 24 & \\
\hline & 00 & 59 & 900 & 17 & 876 & 9.13 & 9.09 & 936 & 9.21 & .68 & 823 & 305 & 774 & 941 & 9.51 & 905 & 830 & $-7 \%$ & 7.17 & 768 & 829 & 843 & 782 & 876 & 15 & 925 & 78 & 8.15 & 67 & $-77<$ & 52 & \\
\hline & 00 & 879 & -897 & -1.19 & 8.85 & -9.21 & 907 & 937 & 913 & 60 & 820 & 19 & -7.78 & 9.15 & 945 & $8 \mathrm{es}$ & 630 & 780 & 726 & 74 & 847 & 347 & 173 & 889 & 835 & 89 & 30 & 725 & 50 & 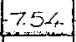 & 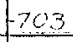 & \\
\hline $7 / 3$ & $\infty$ & 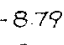 & -9.19 & 19 & 689 & 9.25 & 9.10 & $-9<5$ & -9.16 & 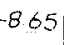 & 820 & 08 & .780 & 44 & $\dot{\phi o}$ & 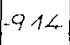 & 827 & $77 !$ & 13 & 76 & 832 & 947 & -779 & 89 & 18 & 83 & 354 & 804 & 59 & 765 & 4 & \\
\hline ." & & 9.11 & -977 & 129 & -920 & -9.30 & $9 \leq 2$ & -9.56 & 945 & 97 & 831 & 820 & -788 & 50 & -0.97 & 933 & 867 & 775 & 50 & $7 z$ & & 854 & $\geq 92$ & 039 & 962 & 19 & 826 & $=8+4$ & 55 & 12 & 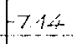 & \\
\hline " & 00 & 1007 & 1037 & -1.43 & 092 & -092 & -053 & $10 \leqslant 7$ & 950 & 22 & 9.10 & -830 & 836 & 169 & 1130 & 1052 & 921 & 805 & 779 & 795 & -895 & 93 & 846 & 901 & 0.54 & 1089 & 029 & 083 & 57 & & 78 & \\
\hline 11 & .00 & 9.91 & 1073 & 140 & 982. & 1025 & 0.02 & 1030 & 9.76 & 938 & 0.24 & -024 & 858 & 1192. & 115 & 1052 & 925 & 39 & 858 & 34 & 032 & 9.3 & 87 & 994 & 111 & 1063 & 85 & 948 & 10 & 02 & 3,3 & 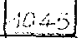 \\
\hline $25 / 3$ & 00 & 10.15 & -1079 & $-1 ; \theta$ & 1024 & -1027 & 1007 & -10.35 & 10.05 & -9.48 & 918 & -927 & -981 & $=12.10$ & $\begin{array}{lll}11 & 82\end{array}$ & 10.73 & 939 & -903 & 880 & as. & -9. & 95 & 88 & 10 & 42 & 979 & 008 & 98 & 37 & 11 & 314 & \\
\hline 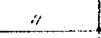 & bo & 10.19 & 1087 & $1<5$ & 1035 & -1038 & -1055 & $10>7$ & 1049 & 98 & 9.97 & -9.45 & un & 1180 & $\mid 11+7$ & -1075 & -974 & -904 & 8.09 & 2 & 4.4 & 927 & -844 & $-100 ?$ & $1+11$ & 1095 & 107 & 107 & 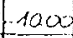 & 1 & 40 & \\
\hline$"$ & 00 & 10.32 & -1102 & 1.56 & 1070 & 1073 & 1063 & -1105 & 1076 & -10.10 & 0.030 & $=935$ & -868 & $1-193$ & $11.8+$ & 145 & 284 & 93 & 885 & 867 & 1950 & 930 & 1356 & 1047 & $11<2$ & nom & 05 & sc & 40 & 22, & 36 & \\
\hline $9 !$ & 100 & 10.50 & 12 & -1.05 & 1073 & -1070 & 103 & 1.107 & 0.82 & 25 & 10 & 1008 & -8.98 & -1210 & 1207 & 1096 & $\log 9$ & .956 & 921 & $100 ?$ & 1010 & $-9 \leq \leq 1$ & 852 & 1018 & -1139 & 09 & & $291\}$ & 30 & 27 & 58 & \\
\hline 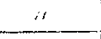 & & 1023 & 75 & -1.53 & -10.39 & 1075 & 272 & -1102 & 774 & $\Delta x$ & $-100 ?$ & 0. & 33 & 1.92 & 198: & & 9.30 & .936 & 9.38 & $8 \varepsilon_{4}$ & -0.59 & 954 & 39 & 1005 & 1072 & 59 & & Los & 872 & 852 & 18 & \\
\hline$\because$ & & 1050 & 10.74 & 1.01 & $10 \times 4$ & $=1054$ & 1075 & 1115 & 10.87 & 1029 & 1022 & 1008 & 918 & 2.16 & 1215 & 1104 & 1007 & 961 & $9 \div 8$ & 201 & .987 & 9.79 & 0.12 & $105 \%$ & 11.09 & 122 & $10 x$ & 981 & 273 & 52 & 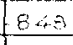 & \\
\hline 301 & & 10.59 & -1097 & 162 & $-10<5$ & 1088 & 1400 & 1417 & -1111 & -10.85 & -10.42 & 5 & -9.32 & & 12.52 & 1090 & -1037 & $10: 9$ & 9.71 & 90 & 1020 & 100 & -914 & -2 & $-11+15$ & $-\infty$ & 104 & -9.9 & 960 & 3 & & \\
\hline 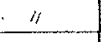 & & 4059 & 1097 & -1.62 & 10.50 & -1108 & 1100 & -1128 & -10.84 & $10 \div 6$ & 1034 & -10.55 & -943 & 1226 & 12.87 & $-14.14 \pi$ & 1037 & 9.72 & 054 & -30 & 1012 & 1029 & 926 & 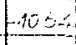 & -11.42 & 11 & $10 \leq$ & -10.51 & 987 & 10 & & \\
\hline$\not$ & & 1059 & -1107 & 162 & $10<5$ & -10.85 & $10.8<$ & -11.17 & 10.91 & -10.45 & 1038 & 10.22 & -9.34 & 1222 & 122.23 & 18 & 10.37 & -966 & $0.9 ?$ & 1927 & -9.95 & $190 \leq$ & .020 & $105:$ & -420 & $-10+5$ & 37 & -1003 & .977 & $-0,2$ & 572 & $=$ \\
\hline 3.1 & & -1139 & 57. & 1.62 & 105.5 & 1407 & 1088 & -11.47 & 10.98 & 1083 & -10.32 & -1045 & -9.27 & 1230 & 1229 & 11.49 & 1037 & 9.36 & 991 & -9.24 & -9.94 & -10.14 & 1055 & $-10,5$ & 1132 & $\therefore O 8$ & 104 & 1050 & 994 & $-10 a$ & -873 & \\
\hline " & & 1099 & -1129 & -167 & 1045 & 1030 & 1086 & $-N 47$ & 119 & -10.58 & -103 & -10.50 & -948 & 12.40 & 120 & 11.30 & 104 & 989 & 938 & -8.69 & -9.72 & -974 & 9.77 & 1024 & -1172 & $\operatorname{Enz}$ & 103 & $-10+3$ & -9.82 & -10.52 & 877 & $\frac{d \sqrt{t}}{4}$ \\
\hline "I & 100 & 1087 & H31 & -1.58 & 1045 & 1033 & 10.80 & 1107 & 1109 & \$o.4t & 1034 & 10.46 & 0.33 & 1245 & 1212 & 1120 & 10.37 & 0.68 & -948 & 3.94 & -9.92 & -1997 & 919 & 1081 & 1132 & 091 & 04 & 1034 & 0.90 & 972 & 853 & - \\
\hline 14 & yo & 2.69 & 14.45 & 160 & 990 & 1051 & 1000 & 1097 & 1079 & 075 & 1032 & 017 & 928 & 143 & 110 & 10 & 10. & -932 & 923 & 3.5 & -075 & 9.9 & 9.2 & 106 & 12 & 08 & 0.05 & 103 & 0.5 & 927 & $5 \leq 3$ & \\
\hline$"$ & & 1059 & $\because 107$ & 60 & 0.31 & 1008 & $0.61)$ & 10.67 & $10 \% 5$ & -1038 & 1024 & $-10+15$ & 933 & 238 & 1224 & 1105 & 102 & $9.5 \%$ & 938 & 82 & 34 & 9.90 & & 00 & 115 & 8 & $1.10 \%$ & 4 & 95 & 0.0 & 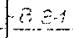 & \\
\hline "I & 0.00 & $\$ 10$ & 1157 & 1.65 & 10.45 & 10.92 & $-108 ;$ & 1123 & 103 & 27.75 & 1055 & 1037 & 9.63 & -1250 & 1245 & 11.50 & 1007 & -9.83 & .960 & 9.07 & 4001 & 100 & 254 & 1111 & 119 & 43 & 1078 & & & 48 & 205 & \\
\hline$\therefore$ & & 1429 & 1164 & -1.68 & 25? & 10.89 & 10.89 & $(-4429)$ & 9.99 & 293 & -1058 & -10.3 & 968 & 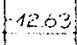 & 1249 & 1154 & 1003 & -975 & 9.50 & .894 & -9.78 & 10 & -964 & 11.12 & 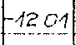 & & 0.8 & & 324 & & 873 & \\
\hline & & 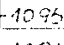 & -1150 & -170 & . & 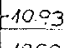 & 1090 & $-11 t^{\prime}$ & $d s$ & 58 & 30 & 1018 & -948 & -122 & 1211 & 4 & -1052 & -9.60 & -952 & 889 & 987 & 100 & $=-9<4$ & -10.97 & -1455 & +110 & 10. & 0 & & & $\theta$ & \\
\hline & 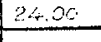 & $10 \%$ & 11.57 & -1.65 & 1029 & $10 \Omega 8$ & 4089 & 119 & 10.95 & 1066 & 1062 & 1026 & -8.55 & 124 & 1211 & 1145 & -1054 & -0.71 & -9.48 & -9.05 & -10.07 & to2? & 9.79 & $-11.1 \alpha_{t}$ & -11.97 & $\therefore 124$ & 10.4 & -1054 & 1020 & & 10 & \\
\hline$=$ & & 12. & 19 & -153 & 01 & 10 & 49 & + & 265 & 4 & -1010 & 8 & & 1 & $-11: 76$ & & 103. & $-9 \leq \leq 3$ & 9.4 & 893 & -9.26 & 1006 & 9.51 & 10.87 & 1106 & 109 & $\therefore 2$ & -9.84 & -9.95 & 2468 & & \\
\hline -." & & & -1112 & & & 10 & & 10 & 6.8 & & 1049 & 1 & 18 & -12.10 & & & -10 & 19.46 & 9.01 & -8.89 & 0.72 & 0990 & -9.42 & -10.87 & 11.58 & -1119 & 1010 & -9.54 & .900 & 9.68 & & \\
\hline$" 1$ & & 10 & 4150 & -165 & 1038 & -16 & 10.63 & -1402 & 10.74 & -10.41 & -1024 & 19.85 & 22 & 1 & 34 & $11+3$ & 1042 & 9.74 & 9.48 & 905 & - 1009 & 1029 & -9.70 & -11.01 & 1190 & $\operatorname{tex} x$ & 1036 & 40 & $F$ & 1012 & 5.10 & \\
\hline 616 & & & & 170 & $\infty x$ & & 93 & & & 74 & & & & & 3 & & & 86 & 9.60 & 9.13 & 70 & 103 & 98 & 1 & 12.0 & 1 & $0 B$ & 10 & 237 & & 26 & \\
\hline - & & 410 & 452 & 75 & 1068 & 1083 & es & -112 & 193 & $=\varepsilon$ & F & 100 & & & 130 & & 10 & $=-1$ & 83 & 62 & 18 & 100 & 71. & 84 & -1475 & 1176 & 07 & 10 & 8 & 8 & 23 & \\
\hline " & 00 & 1111 & $11 \div 02$ & 0.177 & 1050 & 11.02 & -1095 & +1124 & -14.01 & $10 z$ & 1002 & $-10<5$ & 9.78 & -1260 & $12: 34$ & -11.55 & $\begin{array}{l}100 \\
\end{array}$ & -9.84 & 968 & 9.04 & -9.07 & 501 & 597 & 11111 & 1190 & -1187 & 10.54 & 10.00 & 1040 & 97 & 0.17 & \\
\hline $6 / 4$ & $\infty$ & 1132 & 117 & 175 & 1090 & 1133 & 10.85 & $1 \div 54$ & 1121 & 1095 & 1075 & 105 & 988 & -129 & 72 & 179 & & & & & & 105 & 909 & 143 & $\because 22 \pi$ & 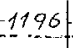 & 10.5 & & 3.3 & 10 & 3 & 24 \\
\hline & & $4+23$ & 12 & 78 & 116 & $5^{-17}$ & 1.2 & 15 & 1.62 & 93 & 0.8 & & 8 & & 589 & 80 & & o & 10. & $1-1$ & -100 & -10.52 & $9.93\}$ & 143 & $\rightarrow$ & & & & & & & \\
\hline 64 & & 1014 & 108 & $0:$ & -1047 & 10.78 & 65 & $10=$ & 1027 & 48. & 25 & 86 & 140 & 118 & 41.25 & 0.70 & $=967$ & 8.93 & 909 & 8.74 & -9.25 & -9.97 & 0,13 & 10 & 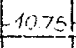 & -- & 0 & 096 & 20 & -9.3 & 83 & \\
\hline & & 100 & 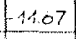 & 80 & $\underline{\theta \in 0}$ & 1114 & -4 & 4. & 14 & & $10 \leq 2$ & 285 & 61 & 119 & 1260 & 70 & 162 & $-9 . \varepsilon_{3}$ & 38 & 4 & $\dot{q}$ & & & & H.E. & 197 & & 1 & & & & \\
\hline & & & & & & & & & & & & & & & & & & & & & & & & & & & & & & & & \\
\hline
\end{tabular}


Soixante cinq pompes ont fonctionné pendant les 14 jours de l'essai, excepté le $26 / 3$, de 16 h 00 jusqu'à 08 h 00 du 27/3, où seulement 38 pompes fonctionnaient ; et le $7 / 4$ (de 09.00 à 21.00 heures) où seulement 45 pompes étaient en fonction.

\subsection{Mesures des niveaux d'eau dans les puits}

Les niveaux d'eau ont été mesurés dans les puits le $26 / 3$ à 12.00 heures), le $27 / 3$ (à 10.00 heures et à 16.00 heures), le $28 / 3$ (à 12.00 heures) et le $10 / 3$ (à 15.00 heures). Toutes les mesures sont données dans le tableau 3.

La figure 15 présente deux coupes du $1^{\text {er }}$ bassin indiquant les niveaux de la nappe à travers les puits et les hauteurs pièzomètriques à la côte - $16 \mathrm{~m}$ des pièzomètres.

Le ler avril après avoir débranché les systèmes d'arrêt et de démarrage et après avoir laissé les 65 pompes fonc- tionner au maximum de leur puissance, nous avons mesuré les niveaux les plus bas, dans chaque puits. Les résultats sont présentés dans la figure 16 .

\subsection{Mesures de débit}

En utilisant un élément de canal (en tôle) rectangulaire, transportable, avec un déservoir triangulaire à l'une extrémité et un raccord pour les tuyaux collecteurs à l'autre, on mesurait le débit de chacun des 21 tuyaux collecteurs, en appliquant la formule de Gourley et Crimp. L'élément de mesure était transporté par une grue et on arrivait à faire les 21 mesures dans une heure. En faisant de nouveau l'hypothèse simplifiante que les mesures étaient faites simultanément on a mesuré des débits de l'ordre de $Q=440 \mathrm{l} / \mathrm{sec}$.

D'autre mesures plus simples (vitesse d'écoulement dans le canal de décharge à la surface libre) ont donné des résultats comparables.

Tableau 3

Mesures des niveaux d'eau dans les puits pendant le pompage

\begin{tabular}{|c|c|c|c|c|c|c|c|c|c|c|c|c|c|c|c|}
\hline DATE & HEURES & 62 & 23 & 67 & 50 & 22 & 51 & 21 & 13 & 53 & 14 & 59 & 15 & 60 & 16 \\
\hline $26 / 3$ & 12.00 & 13,91 & 13,35 & 13,49 & 13,64 & 11,88 & 9,21 & 9,34 & 9,59 & 10,80 & 8,95 & 8,91 & 13,72 & 10,21 & 12,93 \\
\hline $27 / 3$ & 10.00 & 12,71 & 12,75 & 12,09 & 9,49 & 11,08 & 12,86 & 9,19 & 9,39 & 11,15 & 8,95 & 8,86 & 13,97 & 13,31 & 12,43 \\
\hline$"$ & 16.00 & 14,91 & $|13,65|$ & 13,59 & 11,44 & 11,78 & 13,51 & 11,44 & 9,99 & 12,20 & 12,55 & 14,21 & 13,92 & 13,61 & 13,83 \\
\hline $28 / 3$ & - & - & - & - & - & - & - & - & - & - & - & - & - & - & - \\
\hline \multirow[t]{2}{*}{$30 / 3$} & 15.00 & $\mid 1+31$ & 13,65 & 13,79 & $|12,54|$ & 12,43 & 13,21 & 11,54 & 10,19 & 12,40 & 11,85 & 14,41 & 14,07 & 13,91 & 14,23 \\
\hline & & 72 & 43 & 42 & 33 & 71 & 34 & 70 & 51 & 17 & 52 & 18 & 68 & 20 & 69 \\
\hline $26 / 3$ & 12.00 & 13,12 & 10,33 & 9,84 & 11,60 & 10,45 & 13.66 & 8,56 & 12,31 & 13,08 & 11,49 & $|11,66|$ & 7,98 & 12,35 & 10,45 \\
\hline $27 / 3$ & 10.00 & 9,47 & 9,53 & 9,44 & 11,60 & $|10,45|$ & 12,36 & 8,71 & 8,11 & 6,98 & 10,89 & 7,06 & 7,83 & 7,65 & 7,45 \\
\hline 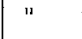 & 16.00 & $11,17 \mid$ & 10,33 & 10,59 & 10,70 & $11,15 \mid$ & 9,86 & 9,16 & 12,46 & 9,18 & 11,69 & 12,36 & 12,13 & 13,05 & 11,65 \\
\hline $28 / 3$ & - & - & - & - & - & - & - & - & - & - & - & - & - & - & - \\
\hline \multirow[t]{2}{*}{$30 / 3$} & $\$ 5.00$ & 13,57 & 10,23 & 10,84 & $11,10 \mid$ & 11,85 & 13,96 & 9,96 & 12,66 & 11,78 & 12,19 & 13,56 & 13,68 & 13,15 & 13,65 \\
\hline & & 1 & 37 & 2 & 38 & 3 & 39 & 4 & 19 & 35 & 41 & 36 & 40 & 74 & 5 \\
\hline $26 / 3$ & 12.00 & 9,88 & 9,88 & 9,67 & 13,17 & a.m. & 13,19 & 8,26 & 11,70 & 12,06 & 1,44 & 11,32 & 11,28 & 7,72 & 13,25 \\
\hline $27 / 3$ & 10.00 & 9,98 & 9,58 & 9,62 & 13,77 & $\mathrm{a} \cdot \mathrm{m}$. & 8,74 & 8,36 & 7,00 & 12,76 & 4,04 & 11,12 & 7,58 & 7,92 & 12,75 \\
\hline$\because$ & 16.00 & 13,08 & 13,88 & 13,37 & 15,07 & 9,76 & 14,24 & 8,81 & 10,65 & 8,91 & 4,59 & 11,72 & 13,38 & 8,47 & 8,55 \\
\hline $28 / 3$ & 12.00 & - & - & - & - & - & - & - & - & 13,06 & 5,94 & 12,22 & 12,68 & 9,02 & 9,15 \\
\hline \multirow[t]{2}{*}{$30 / 3$} & 15.00 & 14,18 & 11,66 & 13,37 & $15,02 \mid$ & 13,16 & 14,29 & 13,16 & 10,40 & 9,51 & 8,34 & 12,37 & 9,28 & 9,22 & 9,05 \\
\hline & & 77 & 47 & 46 & 29 & 76 & 30 & 75 & 73 & 12 & 31 & 45 & 32 & 44 & 65 \\
\hline $26 / 3$ & .00 & 9.77 & 11,49 & 9,62 & 9,67 & 9,39 & 8,98 & 8,37 & 8,02 & 10,69 & 7,76 & 7,57 & 9,98 & 11,41 & 11,71 \\
\hline $27 / 3$ & 10.00 & 9,57 & 11,79 & 9,62 & 9,62 & $9,44\}$ & 11,48 & 8,42 & 7,82 & 10,59 & 8,06 & 7,82 & 10,18 & 10,91 & 7,61 \\
\hline$"$ & 16,00 & $|14,43|$ & 13,39 & 11,07 & 14,22 & $10,09 \mid$ & 11,03 & 9,07 & 12,97 & 11,29 & 8,41 & 13,72 & 10,78 & 11,66 & 12,31 \\
\hline $28 / 3$ & 12.00 & - & - & - & - & - & - & - & 9,22 & 11,49 & 9,16 & 13,57 & 11,48 & 12,21 & 12,41 \\
\hline \multirow[t]{2}{*}{$30 / 3$} & 15.00 & 14,37 & 13,79 & 11,77 & 14,17 & 10,99 & 14,53 & 10,02 & 9,22 & 11,49 & 9,41 & 14,47 & 9,13 & 11,71 & 12,16 \\
\hline & & 63 & 28 & 64 & 48 & 27 & 49 & 26 & 25 & 66 & 24 & 58 & 10 & 54 & 1.1 \\
\hline $26 / 3$ & 12.00 & 9,50 & 9,86 & 13,31 & 9,51 & 12,55 & 8,52 & 13,75 & 7,56 & 7,82 & $9,7 \in$ & 7,47 & 7,30 & 13,31 & 12,54 \\
\hline $27 / 3$ & 10.00 & 9,55 & a.m. & 13,51 & 9,41 & 12,45 & 8,52 & 13,55 & 7,76 & 7,82 & i.m. & 7,57 & 7,45 & 12,81 & 12,64 \\
\hline$"$ & 16.00 & 13,00 & 13,41 & 13,51 & 14,31 & 9,60 & 14,87 & 14,15 & $|13,36|$ & 13,02 & 10,05 & 14,57 & 8,10 & 7,76 & 12,74 \\
\hline $28 / 3$ & 12.00 & - & - & - & - & - & - & - & $11,66 \mid$ & 11,92 & 12,66 & 11,57 & 11,30 & 11,51 & 11,24 \\
\hline \multirow[t]{2}{*}{$30 / 3$} & 15.00 & 14,40 & $\mathrm{a} \cdot \mathrm{m}$. & 13,81 & $|14,21|$ & 13,55 & 14,87 & 14,45 & 12,76 & 13,22 & i.m. & $14,77\}$ & 11,65 & 14,31 & 12,24 \\
\hline & & 6 & 55 & 7 & 56 & 8 & 57 & 9 & & & & & & & \\
\hline $26 / 3$ & 12.00 & 9,03 & 11,75 & 14,03 & 12,78 & 13,65 & 13,53 & 7,89 & & & & & & & \\
\hline $27 / 3$ & 10.00 & 9,13 & 11,70 & 9,33 & 12,18 & 9,05 & 9,43 & 7,84 & & & & & & & \\
\hline$" 1$ & 16.00 & $10,63\}$ & 11,60 & 11,98 & 12,88 & $13,50^{4}$ & 14,03 & 13,54 & & & & & & & \\
\hline $28 / 3$ & - & - & - & - & - & $-\quad$ & - & - & & & & & & & \\
\hline $30 / 3$ & 15.00 & $10,63 \mid$ & 11,25 & 15,03 & 11,98 & 13,55 & 13,23 & 13,64 & & & & & & & \\
\hline
\end{tabular}




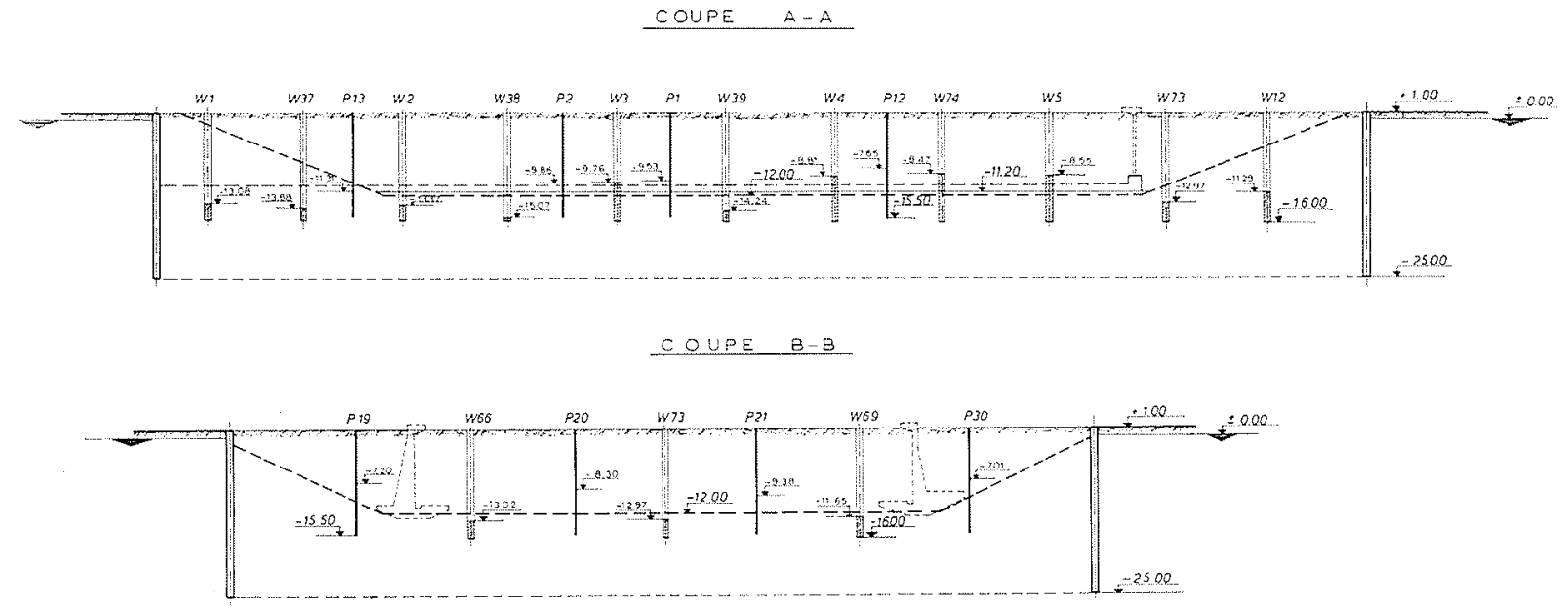

$15 /$ Coupes indiquant les niveaux de la nappe à travers les puits et pièzomètres

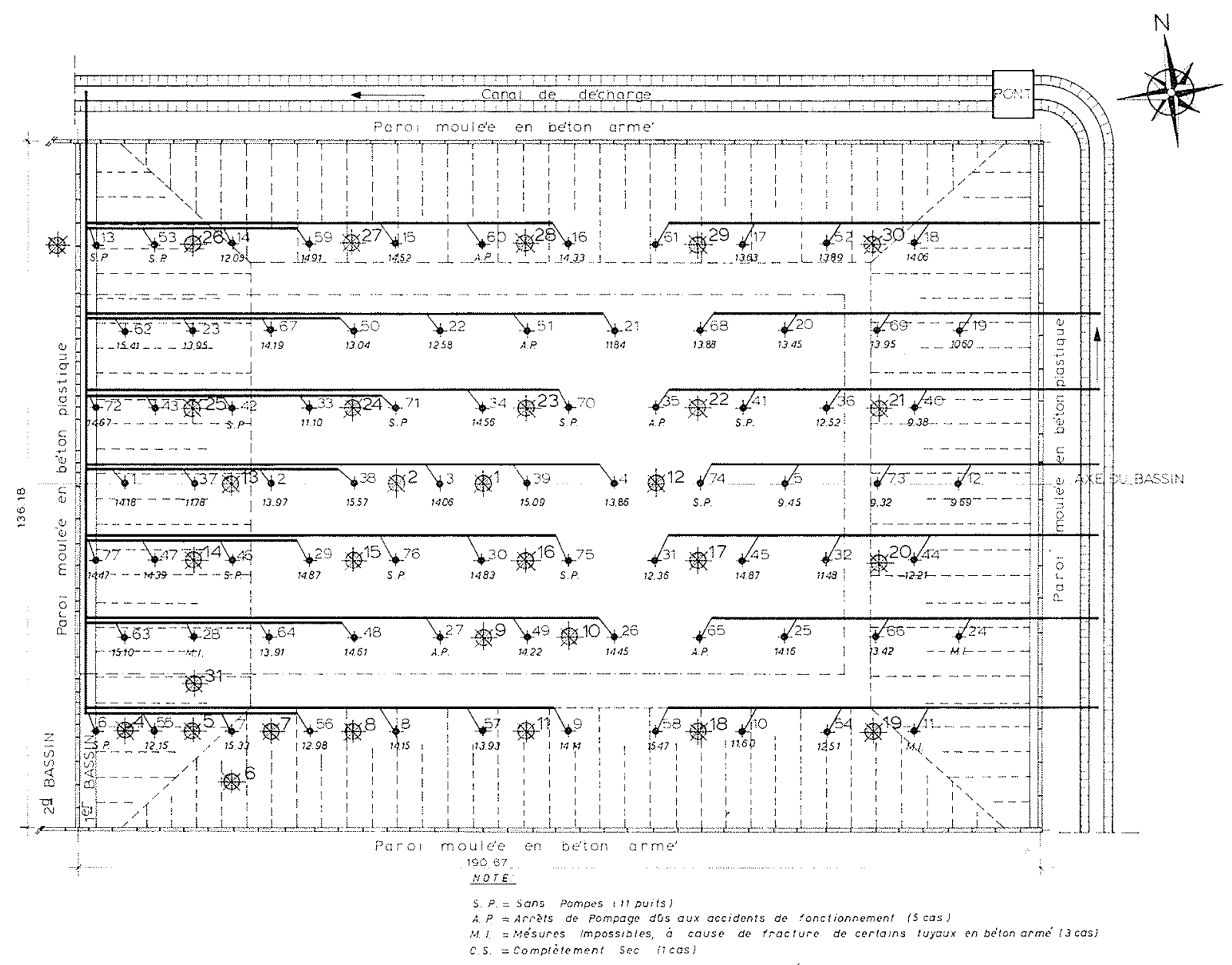

$16 /$ Les niveaux d'eau, les plus bas, mesurés le $1 / 4$ à 14.00 heures

\subsection{Analyses chimiques des eaux}

Des analyses chimiques ont été faites sur des échantillons prélevés à trois positions à des dates différentes (entre le $26 / 3$ et le $5 / 4 / 75$ ). La position était le déchargement des puits $\mathrm{N}^{\circ} 10,11,54$ et 58 et les variations du pourcentage de l'eau de mer est donné dans le graphique (courbe 1) de la figure 17.
Sur la même figure on voit les courbes des positions 2 et 1 avec les numéros des puits correspondants.

Comme l'on peut le noter, le pourcentage d'eau douce diminue considérablement du début à la fin du pompage. Ce phénomène est probablement lié à la présence de la nappe d'eau douce superposée à la nappe d'eau salée ; les parois moulées ont coupé la nappe d'eau douce, et le débit 


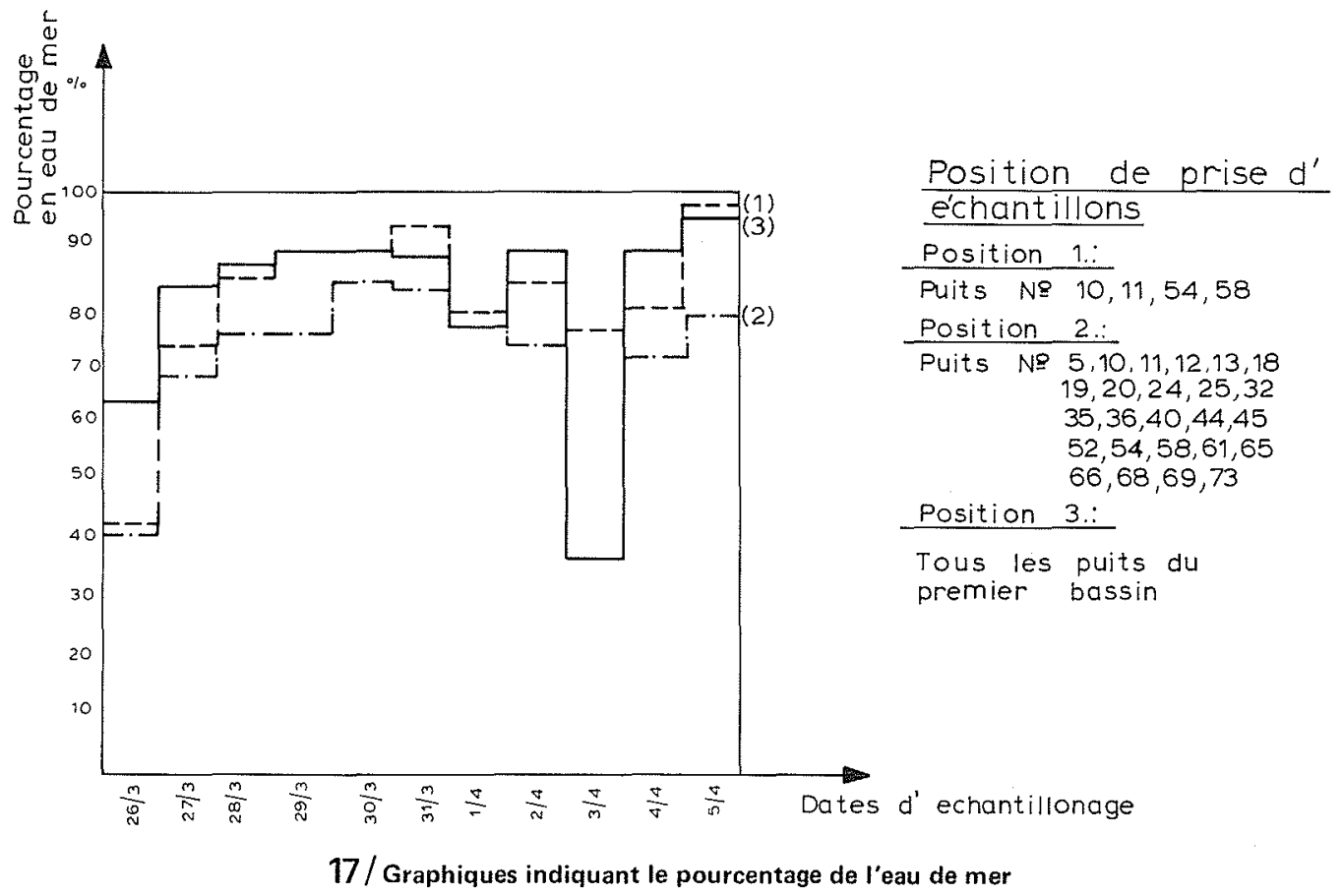

à régime est, pour cette raison, constitué uniquement par les eaux profondes salées.

\subsection{Mesures pendant le retour de la nappe à zéro.}

Le 8 avril à 11.50 heures on a arrêté toutes les pompes. Avant l'arrêt deux groupes de mesures avaient pris place près des deux extrêmités Est et Ouest du bassin d'essai.

Le premier groupe mesurait les niveaux piézomètriques des piézomètres $\mathrm{P} 31, \mathrm{P} 14, \mathrm{P} 25, \mathrm{P} 26$ et les niveaux d'eaux aux puits W55, W28, W37, W23. Le deuxième groupe mesurait les niveaux correspondant aux piézomètres $P 19$, P20, P21, P30 et aux puits W66, W73, W69.

Une personne placée entre les deux groupes donnait le signal de mesurer les niveaux simultanément à tous les points, à des intervalles précis.

Dans le tableau 4 on donne les résultats de ces mesures. Les figures 18 et 19 montrent les variations du niveau d'eau du puits 23 et piézométriques au piézomètre P14.

\section{Conclusions}

\subsection{Rabattement de la nappe}

Les mesures des niveaux piézomètriques et des niveaux d'eau dans les puits, présentés aux tableaux 2 et 3 et aux figures $11,12,13,14,15$ et 16 , prouvent que le niveau de la nappe restait rabattu à la cote de $-12,00 \mathrm{~m}$ environ; le niveau de $-12,00$ était obtenu après quelque jours de pompage. Les premiers jours étaient nécessaires pour la vidange du réservoir formé par les parois moulées. En situation stable on avait atteint un équilibre entre les débits de contournement sous l'écran et de filtration à travers celuici, d'un côté, et le débit pompé de l'autre. La constance du rabattement du niveau à la côte prévue a confirmé les hypothèses de projet et la validité de la solution ancrée, comme méthode constructive de la nouvelle forme de radoub.

Il serait donc possible d'excaver jusqu'à - 11,50 ou - 12.50 et construire à sec les murs et le radier,et ancrer ce radier aux pieux précontraints. Le ler bassin est maintenant excavé. On peut voir sur la figure 20 les têtes des

Tableau 4

Mesures pendant le retour de la nappe à zéro

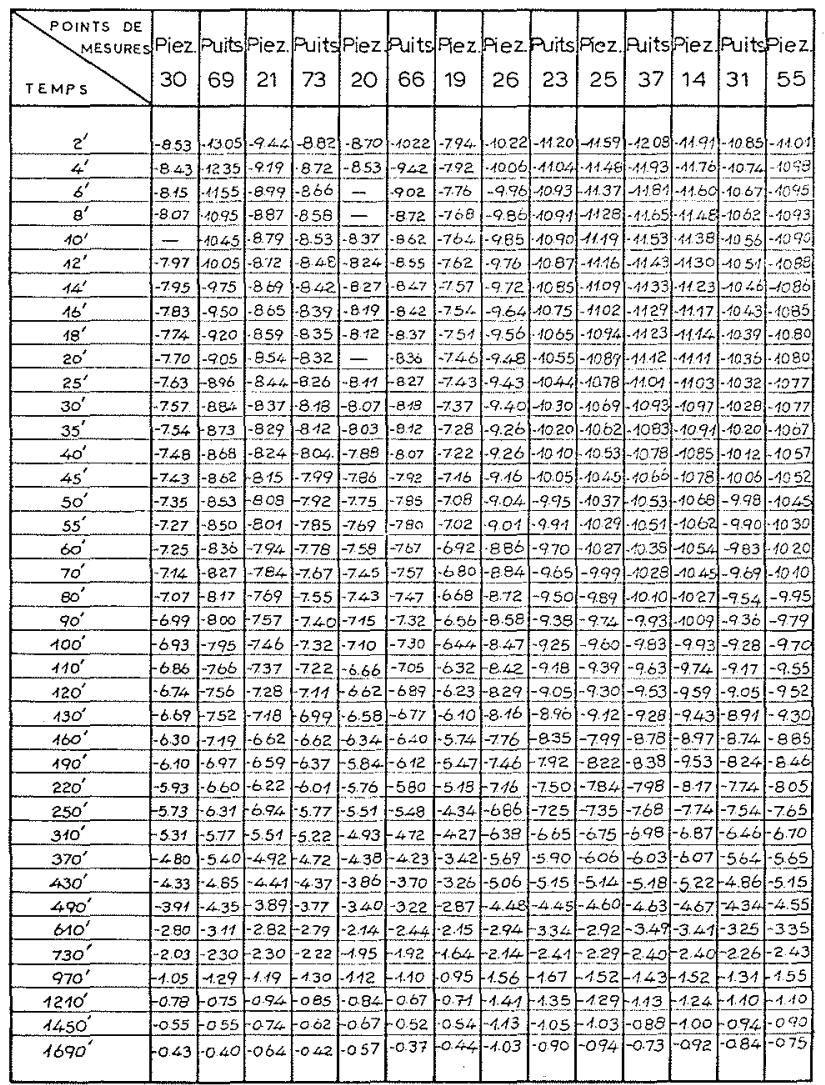




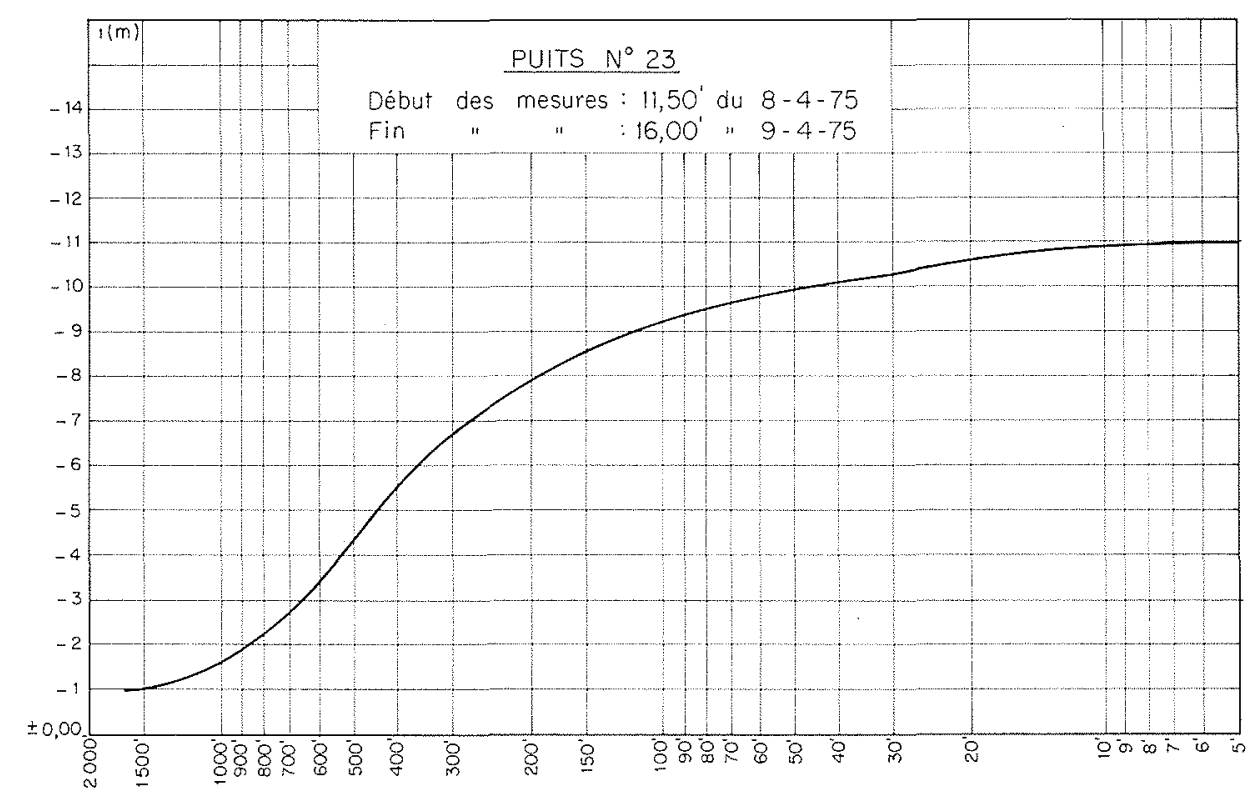

$18 /$ Courbe de relèvement de la nappe au puits $N^{\circ} 23$

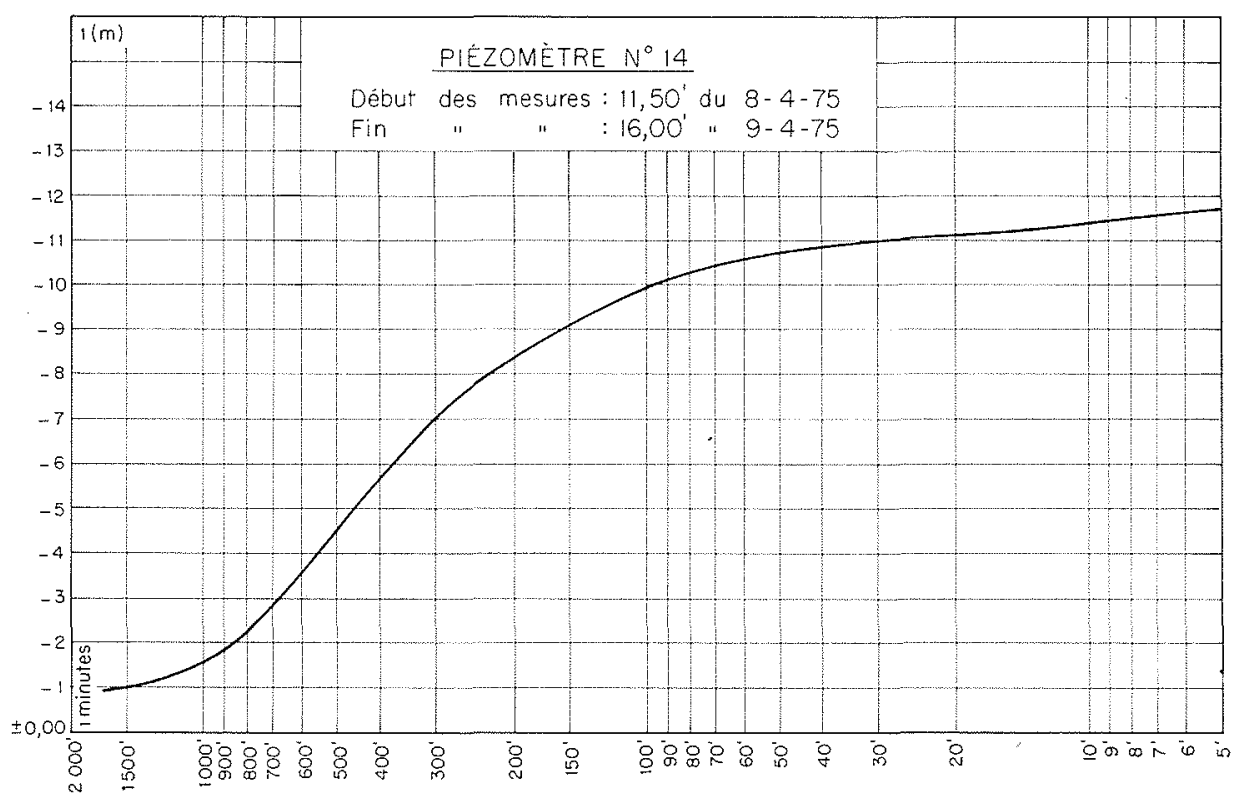

19 / Courbe de relèvement de la nappe au piézomètre $N^{\circ} 14$ 
pieux et panneaux d'ancrage et la préparation du bétonnage de la première partie du radier. A gauche on distingue la tranchée dans laquelle est déjà coulée la fondation de la première partie du bajoyer-nord de la forme de radoub.

\subsection{Débit à pomper}

Le débit calculé théoriquement avant l'essai in situ (valeur maximale de $900 \mathrm{l} / \mathrm{sec}$ et minimale de $400 \mathrm{l} / \mathrm{sec}$ ayant comme moyenne $650 \mathrm{l} / \mathrm{sec}$ approximativement) n'a pas été dépassé durant l'essai (valeur mesurée de l'ordre de $440 \mathrm{l} / \mathrm{sec}$ ). Ceci montre que l'estimation des coefficients de perméabilité à partir des essais faits pendant les recherches géotechniques était correcte, et que les calculs des débits sont presque correspondants à la situation réelle.

\subsection{Qualité de l'eau}

Les analyses chimiques démontrent qu'il n'y a pas de sources d'eau douce en profondeur. On voit bien dans la figure 17 , qu'après avoir pompé l'eau semi-douce du réservoir formé par les parois moulées, il n'y avait que de l'eau de mer à l'intérieur des parois perimétriques. Même les essais des échantillons prélevés aux puits $\mathrm{N}^{\circ} 10,12,19,32$ et 73 , dans lesquels le niveau d'eau restait assez élevé, ont renforcé la conviction que le renouvellement de l'eau du premier bassin était fait par la mer.

Les travaux sont exécutés par la "IMPRESIT-FEDERICI-EDOK-ETER a Joint Venture" sous la surveillance de "T.F. BURNS AND PARTNERS".

Le projet et la consultation technique pour l'exécution ont été confiés aux Sociétés "SOLTEC-GEOPROGETTI".

\section{Bibliographie}

[1]MARTIN (G.P.), IRVINE (K.D.) - Graving dock at Skaramanga, Greece, Proc. Instn. Civ. Engrs, 1972, 52 (Nov) pp. 269 290.

(2) MARTIN (G.P.), IRVINE (K.D.) - Graving dock at Skaramanga, Greece. Discussion, Proc. Instn. Civ. Engrs, 1973, 54 (Aug) pp. 487-494.

[3] ROGAN (A.J.) - The new shipbuilding dry-dock at Sharamanga, Piraeus Technodomica, No. 2, 1975, pp. 35-40, Greece

[4] CARDINAL (J.) DE CAZENOVE (E.) - Ouvrages profonds à radiers perméables. Stations de pompages automatiques. Ecrans d'étanchéité. Reconnaissances et calcul des débits.

[5] MAYER (A.) -Les terrains perméables, Dunod, 1954.

[6] CHAPON (J.) - Travaux maritimes, Eyrolles, 1973.

[7] LAMBE - WHITMANN - Soil Mechanics.

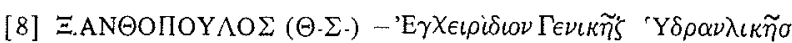

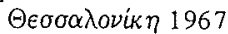

[9] COMOLET (R.) - Mécanique expérimentale des fluides, Masson 1963.

[10]ERNST UND SOHN (W.) - Grundbau Taschenbuch, Berlin, 1966.

[11] SCHNeEbeli (G.) - Les parois moulées dans le sol, Eyrolles, 1972.

[12] VERDEYEN (J.), ROISIN (V.), NUYENS (J.) - Applications de la mécanique des sols, Vander-Dunod, 1971

[13] WASSER -J AHRBUCh 1969 - W. Ernst und Sohn, Berlin 1969

[14] LABAHN (O.), KAMINS KI (W.A.) - Cement Engineer's Handbook, Bauverlag, Wiesbaden 1971.

[15] MARUNG (H.) - Tabellen zur Barechmung von Wasser leitungen, Springer, Berlin, 1957.

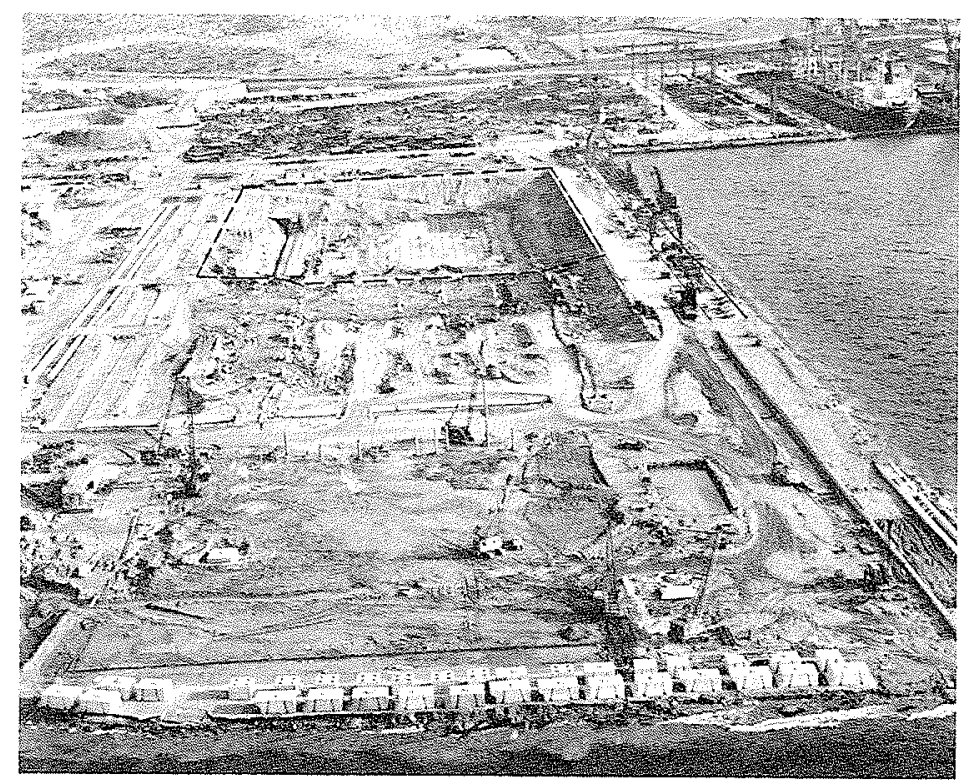

$20 /$ Bassin d'essai excavé $\left(-12,00 \mathrm{~m}\right.$ ) (vue prise le $1^{\text {er }}$ janvier 1976). 